\title{
Composite hot subdwarf binaries - I. The spectroscopically confirmed sdB sample
}

\author{
Joris Vos, ${ }^{1 \star}$ Péter Németh, ${ }^{2,3}$ Maja Vučković, ${ }^{1}$ Roy Østensen ${ }^{4}$ and Steven Parsons ${ }^{5}$ \\ ${ }^{1}$ Instituto de Física y Astronomía, Universidad de Valparaiso, Gran Bretaña 1111, Playa Ancha, Valparaíso 2360102, Chile \\ ${ }^{2}$ Astroserverorg, 8533 Malomsok, Hungary \\ ${ }^{3}$ Dr Karl-Remeis Observatory and ECAP, Astronomical Institute, F.-A.-U. Erlangen-Nürnberg, 96049 Bamberg, Germany \\ ${ }^{4}$ Department of Physics, Astronomy, and Materials Science, Missouri State University, Springfield, MO 65804, USA \\ ${ }^{5}$ Department of Physics and Astronomy, University of Sheffield, Sheffield, S3 7RH
}

Accepted 2017 August 22. Received 2017 August 21; in original form 2017 May 23

\begin{abstract}
Hot subdwarf-B (sdB) stars in long-period binaries are found to be on eccentric orbits, even though current binary-evolution theory predicts that these objects are circularized before the onset of Roche lobe overflow (RLOF). To increase our understanding of binary interaction processes during the RLOF phase, we started a long-term observing campaign to study wide $\mathrm{sdB}$ binaries. In this paper, we present a sample of composite binary sdBs, and the results of the spectral analysis of nine such systems. The grid search in stellar parameters (GSSP) code is used to derive atmospheric parameters for the cool companions. To cross-check our results and also to characterize the hot subdwarfs, we used the independent XTGRID code, which employs TLuSTY non-local thermodynamic equilibrium models to derive atmospheric parameters for the sdB component and PHOENIX synthetic spectra for the cool companions. The independent GSSP and XTGRID codes are found to show good agreement for three test systems that have atmospheric parameters available in the literature. Based on the rotational velocity of the companions, we make an estimate for the mass accreted during the RLOF phase and the minimum duration of that phase. We find that the mass transfer to the companion is minimal during the subdwarf formation.
\end{abstract}

Key words: stars: abundances - binaries: spectroscopic-stars: fundamental parametersstars: horizontal branch - stars: mass-loss - subdwarfs.

\section{INTRODUCTION}

Hot subdwarf-B (sdB) stars are core-helium-burning stars with a very thin hydrogen envelope $\left(M_{\mathrm{H}}<0.02 \mathrm{M}_{\odot}\right)$, and a mass close to the core-helium-flash mass $\sim 0.47 \mathrm{M}_{\odot}$ (Saffer et al. 1994; Brassard et al. 2001). These hot subdwarfs are found in all galactic populations, and they are the main source for the ultraviolet (UV) upturn in early-type galaxies (Green, Schmidt \& Liebert 1986; Greggio \& Renzini 1990; Brown et al. 1997). Furthermore, their photospheric chemical composition is governed by diffusion processes that cause strong He depletion and other chemical peculiarities (Heber 1998). The formation of these extreme horizontal branch (EHB) objects is still puzzling. To form an sdB star, its progenitor needs to lose its hydrogen envelope almost completely before reaching the tip of the red giant branch (RGB), so that the He core ignites while the remaining hydrogen envelope is not massive enough to sustain hydrogen-shell burning. A variety of possible formation channels have been proposed. Currently, there is a consensus that $\mathrm{sdB}$

${ }^{\star}$ E-mail: joris.vos@uv.cl stars are only formed in binaries. Several evolutionary channels have been proposed where binary-interaction physics plays a major role. Close binary systems can be formed in a common-envelope (CE) ejection channel (Paczynski 1976), while stable Roche lobe overflow (RLOF) can produce wide sdB binaries (Han, Tout \& Eggleton 2000; Han et al. 2002). An alternative formation channel forming a single sdB star is the double white-dwarf (WD) merger, where a pair of low-mass He-core white dwarfs spiral in and merge to form a single sdB star (Webbink 1984).

Han et al. (2002, 2003) addressed these three binary-formation mechanisms, and performed binary population synthesis (BPS) studies for two kinds of CE ejection channels, two possible stable RLOF channels and the WD merger channel. The CE ejection channels produce close binaries with periods of $P_{\text {orb }}=0.1-10 \mathrm{~d}$, and main-sequence (MS) or WD companions. The sdB binaries formed through stable RLOF have orbital periods ranging from 10 to $500 \mathrm{~d}$, and MS companions. Chen et al. (2013) revisited the RLOF models of Han et al. (2003) with a more sophisticated treatment of angularmomentum loss. When including atmospheric RLOF, these revised models can reach orbital periods as long as $\sim 1600 \mathrm{~d}$. Finally, the WD merger channel can lead to single sdB stars with a higher mass, 
up to $0.65 \mathrm{M}_{\odot}$. A detailed review of hot subdwarf stars is given by Heber (2016).

Many observational studies have focused on short-period sdB binaries (Koen, Orosz \& Wade 1998; Maxted et al. 2000, 2001; Morales-Rueda et al. 2003; Napiwotzki et al. 2004; Copperwheat et al. 2011), and 148 of these systems are currently known (Kupfer et al. 2015; Kawka et al. 2015). These observed short-period sdB binaries agree very well with the results of BPS studies. Currently, only nine long-period sdB binaries are known (Green, Liebert \& Saffer 2001; Østensen \& Van Winckel 2011, 2012; Barlow et al. 2012; Deca et al. 2012; Vos et al. 2012, 2013; Vos, Østensen \& Van Winckel 2014). Even though this is a small sample, their period-eccentricity distribution shows an unexpected trend. Eight of the nine systems have a significant eccentric orbit, and the entire sample shows a clear correlation of higher eccentricity with longer orbital period (Vos et al. 2015).

Vos et al. (2015) have shown that a combination of two eccentricity pumping mechanisms, phase-dependent RLOF and a circumbinary $(\mathrm{CB})$ disc can explain the observed eccentricities of wide $\mathrm{sdB}$ binaries. However, these models are unable to reproduce the observed trend of higher eccentricities at longer orbital periods. The proposed mechanisms indicate even stronger eccentricity pumping at shorter orbital periods. The two eccentricity pumping processes are highly parametrized, and many of these parameters - such as the mass-loss parameters of Tauris \& van den Heuvel (2006), the $\mathrm{CB}$ disc mass, the redistribution of mass in a $\mathrm{CB}$ disc and many more - are currently unconstrained.

To properly constrain the input parameters of the eccentricity pumping models, a statistically significant sample of well-studied wide $\mathrm{sdB}$ binaries is necessary. In addition to the orbital parameters, the spectroscopically determined properties of the companion star are very relevant to the exploration of any systematic relations between the model parameters and physical reality. The RLOF process in wide $\mathrm{sdB}$ binaries is normally considered to take place on a thermal time-scale, as the sdB progenitor is an RGB star. However, the exact process including mass transfer between the members or mass loss from the system is not well understood. By studying the rotational velocity of the companion, estimates about the accreted mass, and the minimal duration of the RLOF phase can be made. Such estimates are important input in simulations of the RLOF phase.

A comprehensive catalogue containing all currently known hot subdwarf stars has been published by Geier et al. (2017). This catalogue contains $5613 \mathrm{sdO}$ and sdB stars, both single and in binary systems, which have been selected on photometric or spectroscopic criteria.

In this paper, we present a sample of spectroscopically confirmed composite sdB binaries (Section 2) created with the aim to study the binary interaction processes during RLOF. The ongoing longterm observing programme to explore the orbits of composite $\mathrm{sdB}$ binaries is also described (Section 3). Enough spectra have been obtained for nine systems to allow a detailed spectroscopic study of the companions of those wide sdB binaries. The methods employed, as well as a discussion of their accuracy, is given in Section 4, while the atmospheric parameters are given in Section 5 and mass accretion during the RLOF phase is discussed in Section 6. We conclude with a summary and discussion in Section 7.

\section{THE COMPOSITE SUBDWARF-B SAMPLE}

The targets for the current study have been selected from an extensive sample of composite $\mathrm{sdB}$ binaries. This sample is created based on a literature search for spectroscopically confirmed composite $\mathrm{sdB}$ binaries. We required at least medium-resolution spectroscopic observations to include a system as a confirmed composite binary. Most of these targets originated in the surveys of Green et al. (1986), Kilkenny, Heber \& Drilling (1988), Bixler, Bowyer \& Laget (1991), Lamontagne et al. (2000), Aznar Cuadrado \& Jeffery (2001) and Reed \& Stiening (2004). This list of observed systems is supplemented with targets from the Edinburgh-Cape Survey (Stobie et al. 1997), and our own survey sample of UV excess targets from the Galaxy Evolution Explorer (GALEX; Martin et al. 2005) survey, where we selected those stars that had both UV and infrared (IR) excess relative to visual magnitudes.

This sample was constructed independently from the hot subdwarf catalogue of Geier et al. (2017), but it can be seen as a subsample of this catalogue. We have employed more strict selection criteria for a spectroscopic confirmation of the binary systems, rejecting systems that only have low-resolution spectroscopy. Our catalogue contains 10 systems that are not included in the hot subdwarf catalogue of Geier et al. (2017): three systems from various surveys - Ton S 367, KUV 01542-071 and Balloon 82800003 - and seven systems found among the UV excess targets from Galex, J001165423, J02151-7034, J02286-3625, J09000+0128, J17416+6414, $\mathrm{J} 17534-5007$ and $\mathrm{J} 21402-3714$.

In this sample, we have only focused on systems containing an sdB component, and we have excluded all systems with sdO components. The goal of this project is to create a test sample for stable RLOF evolution, of which wide sdB binaries are direct evolution products. Even though sdBs evolve into sdOs, they increase in radius, which can influence the orbital properties, making them less suitable for such a study. Furthermore, there are several known sdO+F/G systems, for example BD- $11^{\circ} 162$ (Østensen \& Van Winckel 2012), which have periods of the order of $100 \mathrm{~d}$. These are most likely remnants of massive stars that lost their envelope on the RGB after non-degenerative He-core ignition. With such massive stars, the envelope is much more tightly bound, and the parameters governing the mass transfer will be very different.

The complete list of confirmed composite sdB binaries from the literature, supplemented with candidates that we have confirmed with our Ultraviolet and Visual Echelle Spectrograph (UVES) observations as described in this paper, comprises 148 systems in total. These are provided as an on-line VizieR catalogue. In Table 1, just the first 10 lines of the complete table are given in order to show the structure. For each system, the coordinates, spectral type and reference are given. The reference refers to the most accurate classification and is provided in the standard 19 digit shorthand used by ADS. When available, we also provide GALEX far-ultraviolet (FUV) and near-ultraviolet (NUV) magnitudes from data reduction 7 , as well as $B, v, g, r$ and $i$ magnitudes from the ninth data reduction of the American Association of Variable Star Observers (AAVSO) Photometric All-Sky Survey (APASS; Henden et al. 2016) and Two-Micron All-Sky Survey (2MASS) $J, H$ and $K_{\mathrm{s}}$ magnitudes (Skrutskie et al. 2006).

For the systems observed with the UVES, the standard classification system for hot subdwarfs is used (see Moehler et al. 1990). Subdwarfs with broad Balmer lines and weak $\mathrm{He}_{\mathrm{I}}$ lines are classified as sdB; if the He II $\lambda 4686 \AA$ line is also visible, they are classified as sdOB. If the systems were analysed with the grid search in stellar parameters GSSP code (Tkachenko 2015) or XTGRID (Németh, Kawka \& Vennes 2012), then the exact spectral type of the companion is given; otherwise, they are indicated as MS. The systems called Jnnnnn \pm nnnn are GALEX targets where the $J$ number is shorthand for the full GALEX identification. 
Table 1. Extract from the VizieR catalogue containing our sample of spectroscopically confirmed composite sdB binaries. The name is the Simbad resolvable name or GALEX number. The reference is given in standard 19 digit format recognizable by ADS. In the VizieR catalogue, seven photometric measurements are given: GALEX FUV and NUV, Johnson $B$ and $v$ from APASS and 2MASS $J, H$ and $K$. Errors on the photometric measurements are denoted by 'e_'.

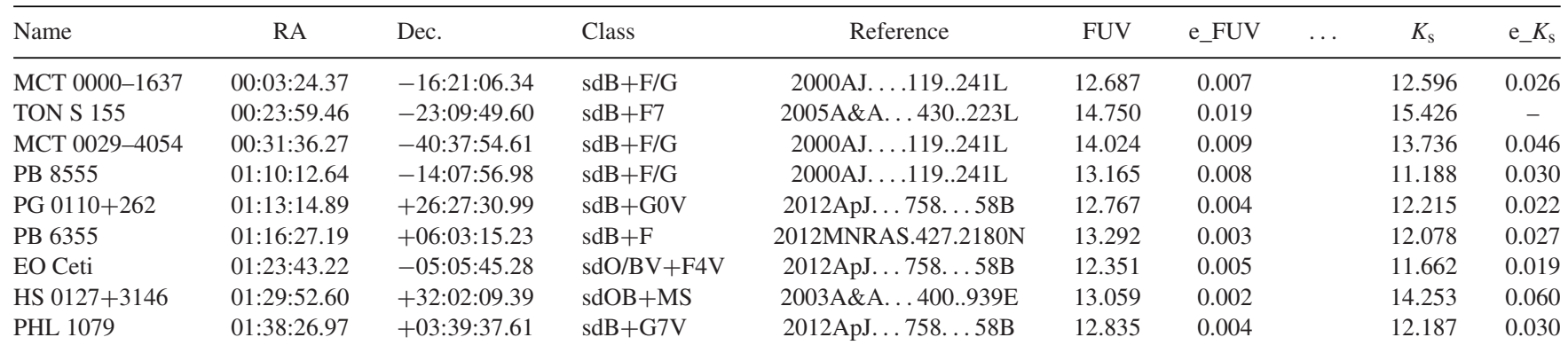

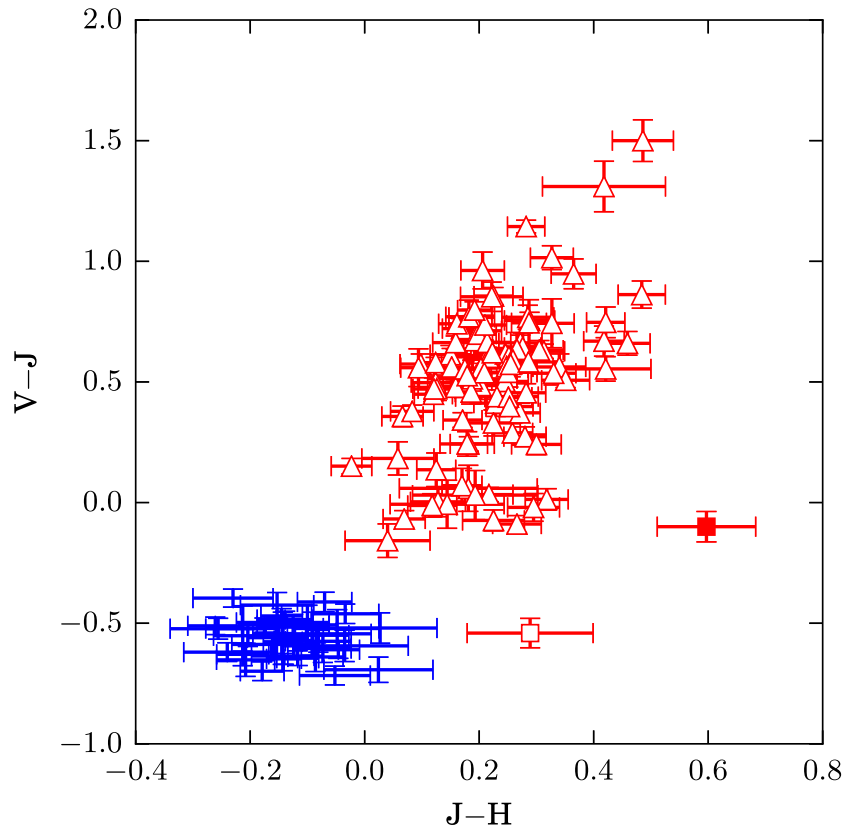

Figure 1. Colour-colour diagram of the composite sdB sample of 2MASS $J-H$ versus Johnson $v-2$ MASS $J$. The composite sdB binaries from the sample presented in this paper are shown as red triangles and, for comparison, the $\mathrm{sdB}+\mathrm{WD}$ binaries of Kupfer et al. (2015) are plotted as blue dots. The two systems that fall outside the main group of composite sdB binaries are PG $1647+056$ (open red square), which is an sdB+K-type binary, and MCT 2340-2806 (filled red square), which is an sdB+G/F-type binary. Both are spectroscopically confirmed.

The systems included in this sample have a magnitude range of $v=10-16 \mathrm{mag}$. In Fig. 1, a $v-J$ versus $J-H$ colourcolour diagram of the composite sdB sample is plotted, together with the $\mathrm{sdB}+\mathrm{WD}$ systems from the short-period sdB binary sample of Kupfer et al. (2015). Only systems with reliable photometric measurements (errors lower than $0.1 \mathrm{mag}$ ) are included in the plot. Both samples are clearly separated in the colour-colour diagram, with the binaries containing an MS component showing significant IR excess compared to the binaries with a WD companion. This division is known (e.g. Green et al. 2008), and has been used to select suitable targets from the GALEX and Edinburgh-Cape blue object surveys. The two composite sdB systems that do not fall within the main body of composite sdB binaries in Fig. 1 are PG $1647+056$, a spectroscopically confirmed $\mathrm{sdB}+\mathrm{K}$ binary (Maxted et al. 2001), and MCT 2340-2806, a spectroscopically confirmed $\mathrm{sdB}+\mathrm{G} / \mathrm{F}$ binary (Lamontagne et al. 2000).

\subsection{Misclassified targets}

Based on the UVES observations, it was found that some targets from earlier IR excess based surveys have been misclassified as composite sdB binaries. These are briefly discussed as follows.

J02292-3959 (= GSC 07552-00389) is a recently discovered cataclysmic variable star of the VY Scl class (Hümmerich, Bernhard \& Srdoc 2014), which shows dramatic and deep (3-4 mag) fading events that can last for several years, related to variations in the mass-transfer rate. Three spectra were obtained over a time-span of two months, but all are remarkably similar. Broad, single-peaked hydrogen and weak helium emission lines are visible in all our spectra originating from the accretion disc surrounding the WD. However, blueward of the $\mathrm{H} \beta$ line, the WD dominates the spectrum (the wings of the Balmer absorption lines are clearly visible), implying that the disc is relatively faint. Thus, our spectra might well have been obtained during a fading event, when the mass-transfer rate is decreased and the disc shrinks and fades. A BLUE arm UVES spectrum of this system is shown in the top panel of Fig. 2.

HE 0505-2806 is a single sdB that shows no variations in radial velocity (RV) within the error bars. However, the spectrum is contaminated by light from a foreground wide binary containing at least one MS F- or G-type star. This contamination caused its misclassification as a composite binary by Vennes, Kawka \& Németh (2011, identified as CD-28 1974). After images taken by the European Southern Observatory (ESO) Faint Object Spectrograph and Camera (EFOSC) at the New Technology Telescope (NTT) in La Silla were acquired, the MS star was clearly visible, and at a distance of 1.35 arcsec from the sdB star. These images were taken at significantly better seeing than our UVES observations, which is why in our observations they appeared unresolved.

EC 12473-3046, classified by Copperwheat et al. (2011) as an $\mathrm{sdB}+\mathrm{MS}$ binary, is likely an sdB binary with an invisible companion. The sdB shows clear $\mathrm{RV}$ variations with a maximum amplitude difference of $\Delta K=27 \pm 3 \mathrm{~km} \mathrm{~s}^{-1}$ in $36 \mathrm{~d}$. The cool companion seems to be stationary within the error on the observations over a period of $2 \mathrm{yr}$. Because of the poor seeing during the UVES observations, it is impossible to distinguish between the sdB binary and the cool star. It is possible that the cool star is bound to the inner binary on a very long period orbit, but in this case it would not have affected the evolution of the sdB. The inner binary must be an sdB with an unseen companion; either a WD or an M-dwarf 


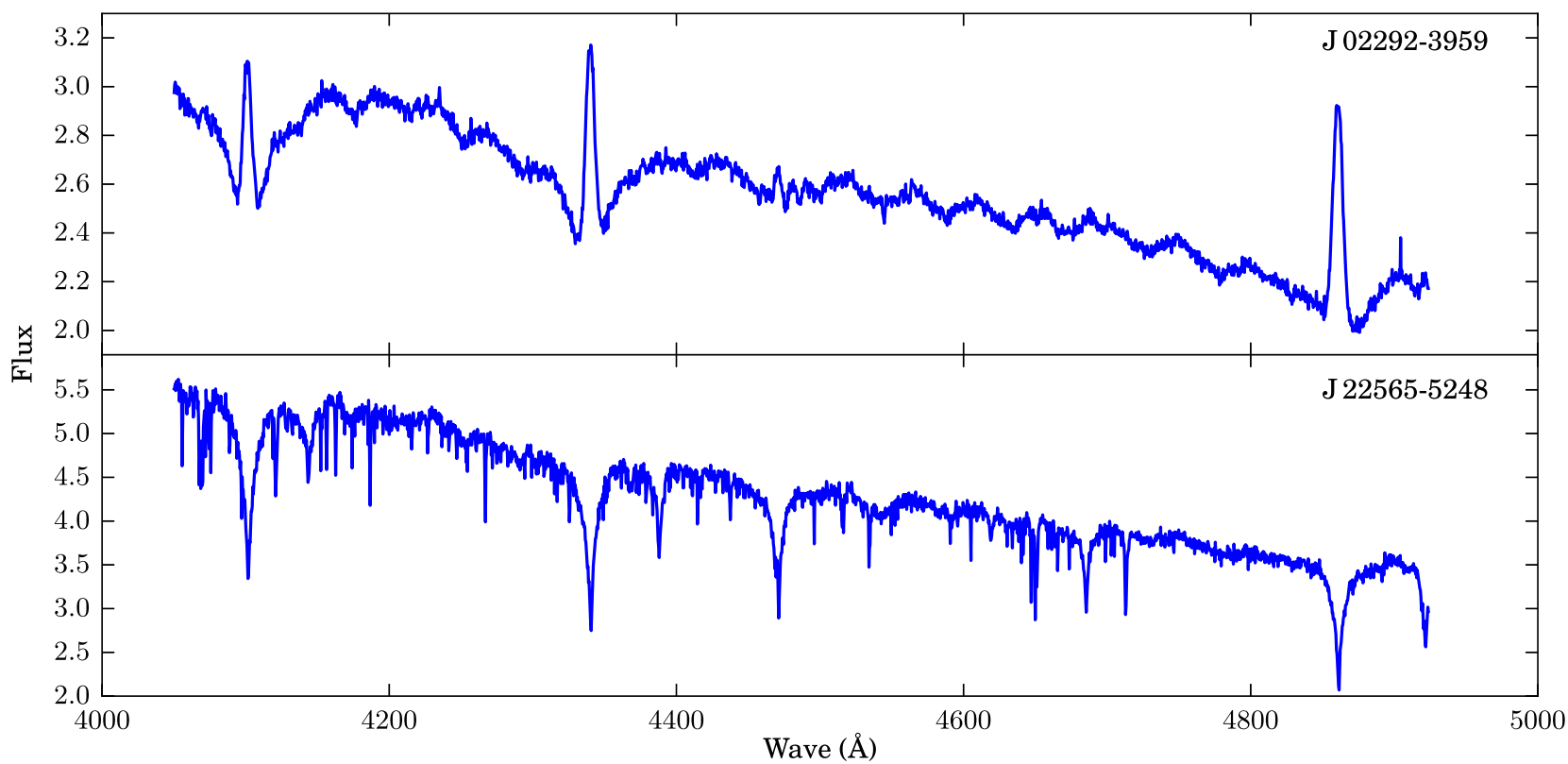

Figure 2. The BLUE arm UVES spectra of the cataclysmic variable J 02292-3959 (top panel) and the He-sdOB J 22565-5248 (bottom panel).

star, making the system a hierarchical triple system. EC 124733046 would be the third candidate for a hierarchical triple system after PG 1253+284 (Heber et al. 2002) and J09510+03475 (Kupfer et al. 2015).

PG $1629+081$ is an sdOB binary with an unseen companion. The amplitude of the RV variations indicates a short-period binary.

J07104+2333 and MCT 2356-2655 are sdO binaries, and as such are not of interest in this paper.

J22565-5248 is a single He-sdOB, which does not show any RV variation. A BLUE arm UVES spectrum of this system is shown in the bottom panel of Fig. 2.

PB 6148 is not a subdwarf star but an F-type star (see also Section 5.3).

Balloon 110607002 was classified as an $\mathrm{sdB}+\mathrm{K}$ system by Bixler et al. (1991), but it does not show any evidence of a subdwarf component in the UVES spectra, and there is no strong UV flux detected by GALEX.

\section{OBSERVATIONS}

Based on our composite sdB sample, explained in Section 2, we initiated a monitoring campaign using UVES at the Very Large Telescope (VLT) Kueyen telescope (formerly UT2) in Cerro Paranal, Chile. UVES is a two-arm cross-dispersed echelle spectrograph, which we have used in standard dichroic- $2437+760$ mode covering a wavelength range of 373-499 $\mathrm{nm}$ in the BLUE arm and 565-946 nm in the RED arm. Using a slit width of 1 arcsec, a resolution of about 40000 was reached. The targets are selected to cover all parts of the sky visible from Paranal, and are brighter than 14 mag. As the orbital period of these systems can be as long as $1300 \mathrm{~d}$ (Vos et al. 2013), observations in six consecutive semesters are necessary to cover the entire orbit. Currently, observations during three semesters, P88 (2011 October 1-2012 March 31), P93 (2014 April 1-2014 September 30) and P96 (2015 October 1-2016 March 31), have been obtained. Thus, no systems have complete orbital coverage yet. However, for 10 systems, enough spectra have been obtained to create a master spectrum suitable for spectroscopic analysis of the cool companion and the sdB. These 10 systems,
Table 2. The number of obtained UVES spectra for each of the 10 targets analysed in this paper and the semester in which each target has been observed. Dates of the semesters are P88 (2011 October 1-2012 March 31), P93 (2014 April 1-2014 September 30) and P96 (2015 October 1-2016 March 31).

\begin{tabular}{lcccc}
\hline Object & \multicolumn{4}{c}{ Number of observations } \\
& P88 & P93 & P96 & Total \\
\hline BPS CS 22890-74 & 0 & 3 & 0 & 3 \\
HE 0430-2457 & 4 & 3 & 2 & 9 \\
HE0505-2806 & 0 & 3 & 0 & 3 \\
J02286-3625 & 0 & 3 & 3 & 6 \\
J03154-5934 & 0 & 3 & 3 & 6 \\
J03582-3609 & 0 & 3 & 3 & 6 \\
JL 277 & 2 & 1 & 3 & 6 \\
MCT 0146-2651 & 0 & 3 & 1 & 4 \\
PB 6148 & 0 & 2 & 1 & 3 \\
PG 1514+034 & 0 & 4 & 0 & 4 \\
\hline
\end{tabular}

together with the number of observations and the semesters in which they are observed, are given in Table 2. All UVES spectra were reduced using the UVES pipeline and the REFLEX workflow engine (Freudling et al. 2013).

To check the methods used to derive the spectral parameters of the cool companions of the UVES systems, three well-studied long-period sdB+MS binaries (i.e. Feige $87, \mathrm{BD}+34^{\circ} 1543$ and $\left.\mathrm{BD}+29^{\circ} 3070\right)$ are included. These three systems, which we refer to as the test systems, have been analysed in detail by Vos et al. (2013). The test systems have been observed with the HERMES spectrograph attached to the 1.2-m Mercator telescope in La Palma, Spain. Furthermore, there are low-resolution $(R \approx 550)$ flux-calibrated spectra of all three systems obtained with the Boller and Chivens (B\&C) spectrograph attached to the University of Arizona's 2.3-m Bok telescope located at Kitt Peak. For BD $+34^{\circ} 1543$, there is also a low-resolution $(R \approx 1800)$ spectrum available from the Large Sky Area Multi-Object Fiber Spectroscopic Telescope (LAMOST) data release 1 (Luo et al. 2016). These low-resolution spectra were analysed with the XTGRID code. 


\section{METHODS}

Spectral parameters for both the sdB and the cool companion are derived using two different approaches. The GSSP software package is used to determine the spectral parameters of the cool companion, using a master spectrum shifted to the rest frame of the companion. The code XTGRID is used to derive spectral parameters for both the $\mathrm{sdB}$ and the cool companion, using for each target the spectrum with the highest signal-to-noise ratio $(\mathrm{S} / \mathrm{N})$. In the following subsections, both methods are described and their accuracy and precision are discussed. Furthermore, three test systems are analysed using GSSP and XTGRID, and the results are compared with values from the literature.

GSSP derives spectral parameters for the cool companion from a master spectrum without removing the contribution of the $\mathrm{sdB}$ companion. This latter contribution is handled by introducing a dilution parameter, and is in fact assumed to be non-variable over the wavelength range considered in the GSSP analysis. This approach might seem counter-intuitive compared to using a spectral model to subtract the $\mathrm{sdB}$ contribution, as is used in, for example, the versatile wavelength analysis (VwA) code. However, here we show that the approach used in GSSP is valid over short spectral ranges where the $\mathrm{sdB}$ contribution is approximately constant.

\subsection{Master spectrum creation}

To create the master spectrum for the cool companion, all spectra are shifted to the rest frame of the cool companion, after which they are summed up and normalized. To determine the radial velocities of the cool companion, a cross-correlation with a template spectrum was performed. The cross-correlation is performed in velocity space, using the RED arm spectrum on the spectral regions 5940-6270 and 6320-6530 A. The template is a synthetic spectrum based on estimated parameters and calculated with the SYNTHV local thermodynamic equilibrium (LTE) based radiative transfer code (Tsymbal 1996). When the GSSP parameters are determined, a new template spectrum is calculated using the derived parameters, and the master spectrum creation and GSSP analysis is repeated. There can be a little smearing of the spectral lines because of the error on the RV measurements, but as the errors on the RVs of the cool companion are of the order of $0.5 \mathrm{~km} \mathrm{~s}^{-1}$, this is negligible. The normalization is done by manually selecting continuum points in the spectrum and fitting a spline function through those points. The resulting master spectra have $\mathrm{S} / \mathrm{N}$ of around 100 .

\subsection{GSSP}

The GSSP software package (Tkachenko 2015) is based on a grid search in the fundamental atmospheric parameters and (optionally) individual chemical abundances of the star (or binary stellar components) in question. It uses the method of atmosphere models and spectrum synthesis, which assumes a comparison of the observations with each theoretical spectrum from the grid. For the calculation of synthetic spectra, we use the SYNTHV LTE-based radiative transfer code (Tsymbal 1996) and a grid of atmosphere models pre-computed with the LLMODELS code (Shulyak et al. 2004).

GSSP allows for optimization of six stellar parameters at a time: effective temperature $\left(T_{\text {eff }}\right)$, surface gravity $(\log g)$, metallicity $([\mathrm{Fe} / \mathrm{H}])$, microturbulent velocity $\left(v_{\text {micro }}\right)$, projected rotational velocity $\left(v_{\mathrm{r}} \sin i\right)$ and the dilution of the star, which is defined as $d=F_{\mathrm{MS}} /\left(F_{\mathrm{MS}}+F_{\mathrm{sdB}}\right)$. The synthetic spectra can be computed in any number of wavelength ranges, and each considered spectral
Table 3. The parameter ranges of the model grid used in the Gssp fit together with the step size. The microturbulent velocity is kept fixed, and $v_{\mathrm{r}} \sin i$ is the projected rotational velocity.

\begin{tabular}{llc}
\hline Parameter & \multicolumn{1}{c}{ Range } & Step \\
\hline$T_{\text {eff }}(\mathrm{K})$ & $3000-9000$ & 100 \\
$\log g(\mathrm{dex})$ & $2.5-4.5$ & 0.1 \\
{$[\mathrm{Fe} / \mathrm{H}](\mathrm{dex})$} & $-0.8-0.8$ & 0.1 \\
$v_{\text {micro }}\left(\mathrm{km} \mathrm{s}^{-1}\right)$ & 2.0 & - \\
$v_{\mathrm{r}} \sin i\left(\mathrm{~km} \mathrm{~s}^{-1}\right)$ & $1-150$ & 1 \\
Dilution & $0.01-1.00$ & 0.03 \\
\hline
\end{tabular}

interval can be from a few $\AA$ up to a few thousand $\AA$ wide. The grid of theoretical spectra is built from all possible combinations of the above-mentioned parameters. Each spectrum from the grid is compared to the observed spectrum of the star and the chi-square merit function is used to judge the goodness of fit. The code delivers the set of best-fitting parameters, the corresponding synthetic spectrum and the chi-square values for each grid point.

For the systems discussed in this paper, GSSP is used to fit the wavelength ranges of 5910-6270 and 6330-6510 $\AA$ in the RED arm of UVES. Because the sdB star is most strongly contributing in the UV and in the blue, these two ranges give the best compromise between a high $\mathrm{S} / \mathrm{N}$ and a high contribution of the cool companion. There are also no spectral lines of the sdB component visible in these wavelength ranges.

The parameter ranges of the model grid used in GSSP are given in Table 3. In the GSSP fit, the microturbulent velocity is kept fixed at 2.0 as the $\mathrm{S} / \mathrm{N}$ of the spectra is not high enough to accurately determine this parameter. The projected rotational velocity is unconstrained and uses steps of $1 \mathrm{~km} \mathrm{~s}^{-1}$. To determine the parameters of each system, first a coarse grid is used that spans the full range with steps of $\Delta T_{\text {eff }}=500 \mathrm{~K}, \Delta \log g=0.5 \operatorname{dex}, \Delta[\mathrm{Fe} / \mathrm{H}]=0.4 \operatorname{dex}$ and $\Delta$ dilution $=0.1$. In the second run, a smaller ranged grid is calculated with the smallest available step size for each parameter.

Together with the chi-squared value for each grid point, GSSP returns the chi-squared value corresponding to the $1 \sigma$ range. This value is used to determine the $1 \sigma$ error on each parameter. As this chi-square value is determined based on the complete grid, all possible parameter correlations are included in the final error. The important parameter correlations in the case of our stars are discussed in Section 4.3. As a rule of thumb, an estimate of the $3 \sigma$ error is determined as $e_{3 \sigma}=2.2 \times e_{1 \sigma}$. Tkachenko (2015) reports that this error estimate corresponds to results obtained by Markov chain Monte Carlo simulations. The errors reported in this paper are the $1 \sigma$ and $3 \sigma$ errors as described here.

\subsection{GSSP: accuracy and parameter correlations}

To determine the accuracy of the GSSP code, and to gain a better understanding of the different correlations between the five variables, a large detailed model grid spanning the entire parameter range given in Table 3 was calculated for the three test systems: $\mathrm{BD}+34^{\circ} 1543, \mathrm{BD}+29^{\circ} 3070$ and Feige 87 . These three sdB binaries with MS companions have been analysed in detail by Vos et al. (2013), and are therefore ideal test cases for the GSSP software package. The chi-square maps of several parameter combinations are shown in Fig. 3.

Based on these grids, it is clear that there is a strong negative correlation between metallicity and dilution. This correlation is expected, as a decrease in the metallicity in the models would lead 


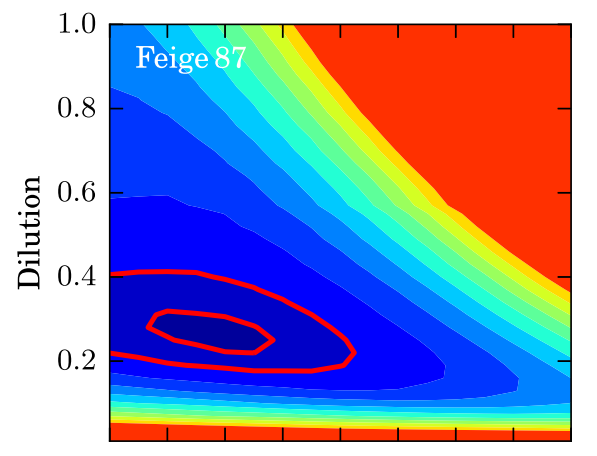

$\begin{array}{lllllll}-0.8-0.6-0.4-0.2 & 0.0 & 0.2 & 0.4 & 0.6 & 0.8\end{array}$
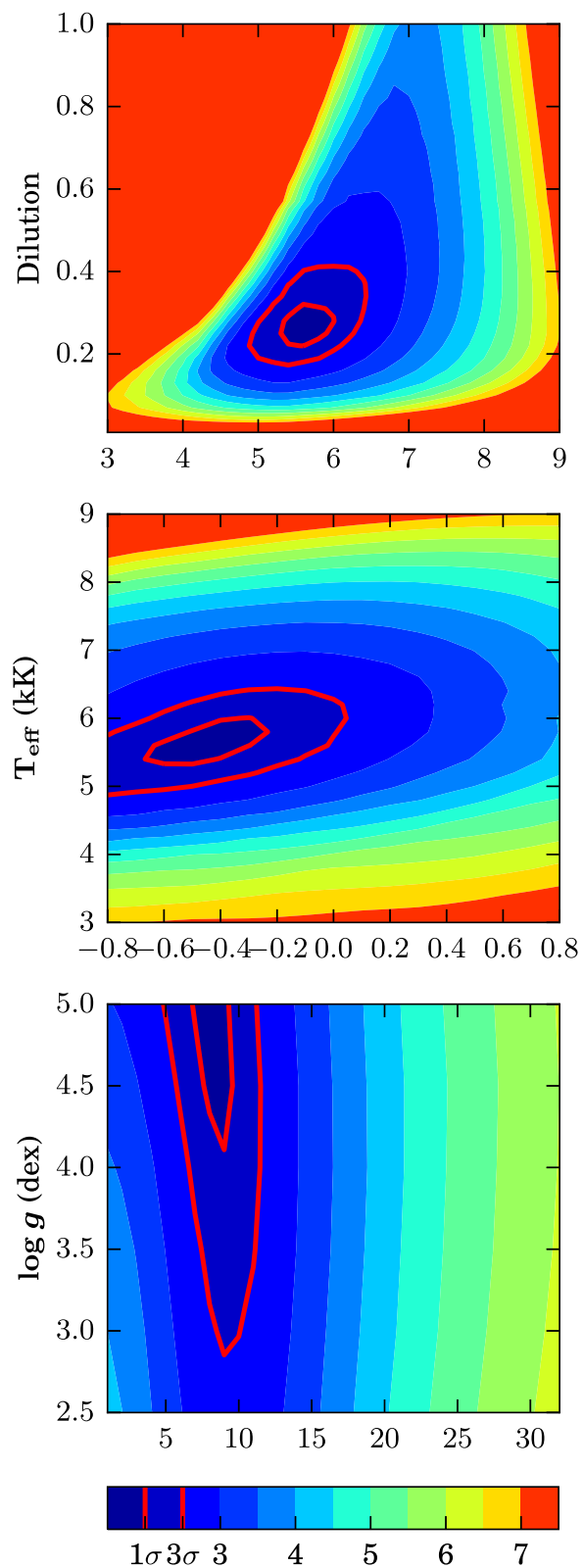
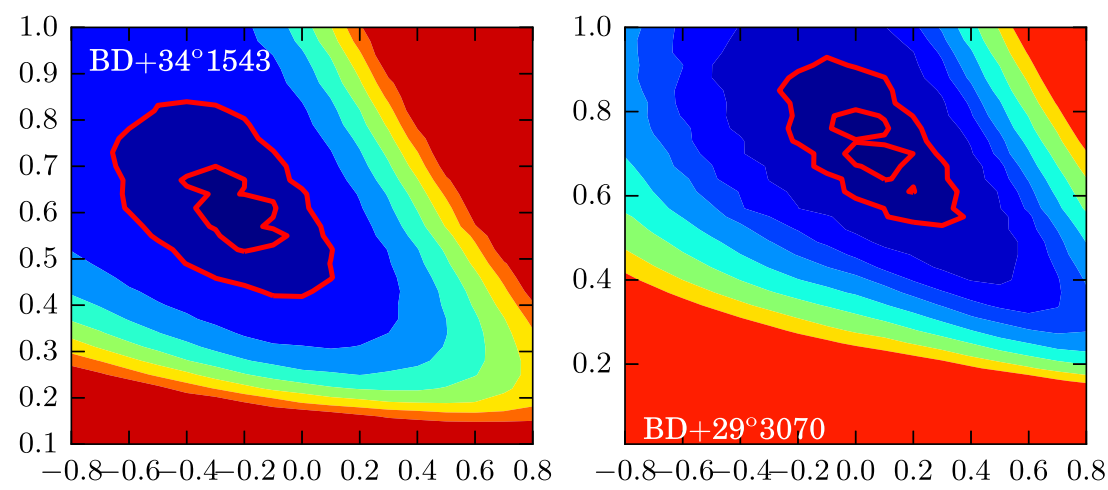

$[\mathrm{Fe} / \mathrm{H}](\mathrm{dex})$
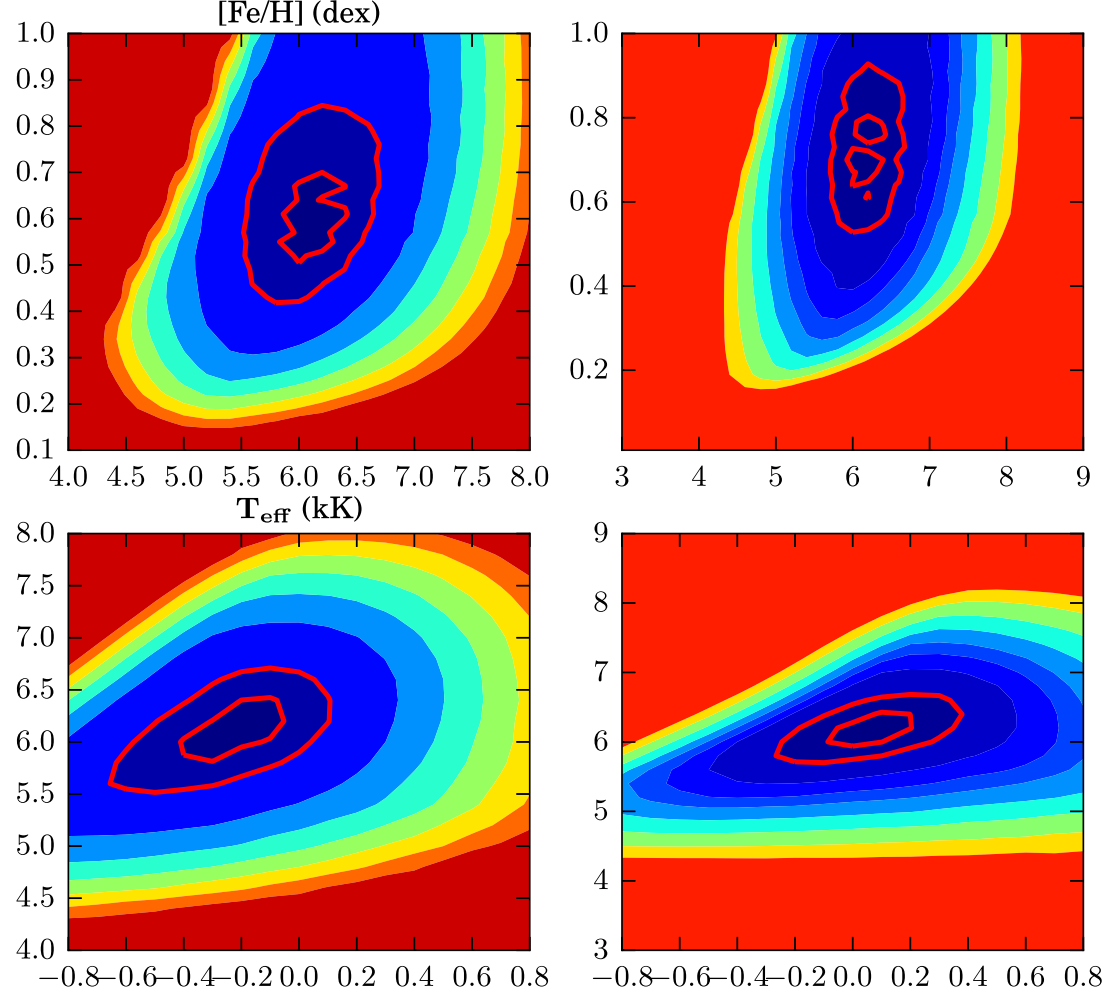

$[\mathrm{Fe} / \mathrm{H}](\mathrm{dex})$

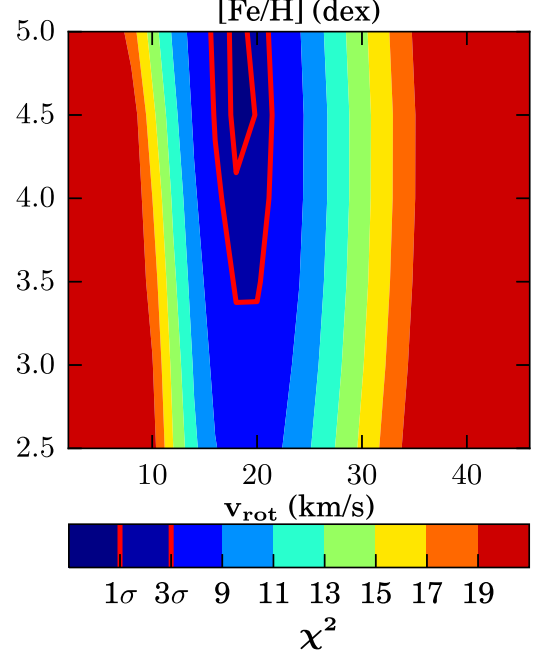

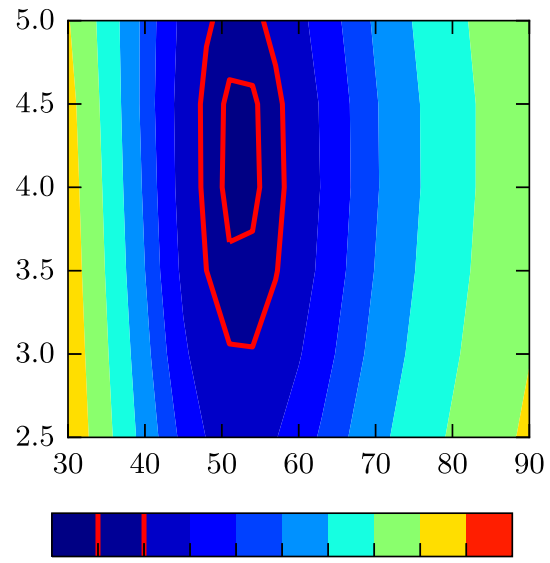

$1 \sigma 3 \sigma 19212326303540$

Figure 3. Chi-squared plots for different parameter combinations for three systems: left, Feige 87 (slow rotator); middle, BD $+34^{\circ} 1543$ (medium rotator); right, $\mathrm{BD}+29^{\circ} 3070$ (fast rotator). The different colours represent different chi-squared values and the $1 \sigma$ and $3 \sigma$ confidence intervals are shown as red lines. See also Section 4.3 . 
Table 4. Comparison of spectroscopic parameters for three wide sdB binaries (BD $+34^{\circ} 1543, \mathrm{BD}+29^{\circ} 3070$ and Feige 87 ) obtained using GSSP and the VWA on high-resolution spectra, using SED fitting based on photometry from the literature and using the XTGRID code on low-resolution spectra. The vwA, SED and XTGRID results are taken from Vos et al. (2013). The dilution is defined as $d=F_{\mathrm{MS}} /\left(F_{\mathrm{MS}}+F_{\mathrm{sdB}}\right)$. See also Section 4.4 .

\begin{tabular}{|c|c|c|c|c|c|c|c|}
\hline & Method & $\begin{array}{l}T_{\text {eff }} \\
(\mathrm{K})\end{array}$ & $\begin{array}{l}\log g \\
(\operatorname{dex})\end{array}$ & $\begin{array}{c}{[\mathrm{Fe} / \mathrm{H}]} \\
(\mathrm{dex})\end{array}$ & $\begin{array}{c}v_{\text {micro }} \\
\left(\mathrm{km} \mathrm{s}^{-1}\right)\end{array}$ & $\begin{array}{c}v_{\mathrm{rot}} \sin i \\
\left(\mathrm{~km} \mathrm{~s}^{-1}\right)\end{array}$ & Dilution \\
\hline \multirow[t]{4}{*}{$\mathrm{BD}+34^{\circ} 1543$} & VWA & $6150 \pm 150$ & $4.20 \pm 0.30$ & $-0.24 \pm 0.12$ & $1.45 \pm 0.25$ & $17 \pm 4$ & - \\
\hline & SED & $6210 \pm 220$ & $4.20 \pm 0.20$ & - & - & - & $0.67 \pm 0.13$ \\
\hline & XTGRID & $6100 \pm 300$ & $4.11 \pm 0.30$ & $-0.40 \pm 0.25$ & - & - & $0.68 \pm 0.10$ \\
\hline & GSSP & $6100 \pm 150$ & $4.50 \pm 0.50$ & $-0.31 \pm 0.10$ & 2.0 & $19 \pm 1$ & $0.60 \pm 0.05$ \\
\hline \multirow[t]{4}{*}{$\mathrm{BD}+29^{\circ} 3070$} & VWA & $6100 \pm 200$ & $4.30 \pm 0.50$ & $0.09 \pm 0.21$ & $1.50 \pm 0.35$ & $52 \pm 4$ & - \\
\hline & SED & $6570 \pm 650$ & $4.40 \pm 0.40$ & - & - & - & $0.72 \pm 0.12$ \\
\hline & XTGRID & $6026 \pm 300$ & $4.26 \pm 0.30$ & $0.08 \pm 0.25$ & - & - & $0.69 \pm 0.10$ \\
\hline & GSSP & $6170 \pm 200$ & $4.30 \pm 0.40$ & $0.05 \pm 0.10$ & 2.0 & $52 \pm 2$ & $0.70 \pm 0.04$ \\
\hline \multirow[t]{4}{*}{ Feige 87} & VWA & $6175 \pm 150$ & $4.50 \pm 0.60$ & $-0.50 \pm 0.25$ & $1.15 \pm 0.25$ & $8 \pm 3$ & - \\
\hline & SED & $5840 \pm 550$ & $4.40 \pm 0.25$ & - & - & - & $0.24 \pm 0.12$ \\
\hline & XTGRID & $5675 \pm 250$ & $4.23 \pm 0.35$ & $-0.60 \pm 0.20$ & - & - & $0.37 \pm 0.10$ \\
\hline & GSSP & $5500 \pm 230$ & $4.50 \pm 0.50$ & $-0.50 \pm 0.15$ & 2.0 & $8 \pm 1$ & $0.30 \pm 0.05$ \\
\hline
\end{tabular}

to weaker absorption lines, which can be countered by increasing the dilution factor. The effect of this correlation can be reduced by using enough different lines that will respond differently on the changing metallicity. Thus, this effect cannot completely be compensated by changing the dilution.

In the slowly rotating Feige $87\left(v_{\text {rot }}=8 \pm 2 \mathrm{~km} \mathrm{~s}^{-1}\right)$, there is also a positive correlation between effective temperature and dilution. This correlation reduces greatly in strength with increasing $v_{\text {rot }}$, and is completely absent in $\mathrm{BD}+29^{\circ} 3070\left(v_{\text {rot }}=52 \pm 3 \mathrm{~km} \mathrm{~s}^{-1}\right)$. The faster rotating systems do show a positive correlation between $T_{\text {eff }}$ and $[\mathrm{Fe} / \mathrm{H}]$, which is almost invisible for Feige 87. This correlation between $T_{\text {eff }}$ and [Fe/H] would - in combination with the correlation between $[\mathrm{Fe} / \mathrm{H}]$ and dilution - cancel out the correlation between $T_{\text {eff }}$ and dilution. This explains why this latter correlation is absent in the faster rotating systems.

Chi-square maps of the test systems for the parameter pairs with the strongest correlations are shown in the first three lines of Fig. 3, where the $1 \sigma$ and $3 \sigma$ confidence intervals are shown as thick red lines. The last line of this figure shows the chi-square maps for $\log g$ versus $v_{\text {rot }}$.

From the $1 \sigma$ and $3 \sigma$ contours shown in Fig. 3, it is clear that most parameters can be determined accurately using the GSSP package. The only exception is the surface gravity. For all three test systems, the $3 \sigma$ range for the surface gravity is about $\log g=[3.0-5.0]$. As the spectral lines in these types of stars have little dependency on the surface gravity, this is expected. For the systems observed with the UVES, which have a lower S/N, the error on $\log g$ will be even larger. The XTGRID code can provide stronger constraints on the surface gravity, as it fits both components simultaneously, and the total flux of the $\mathrm{sdB}$ and cool companion must match the observations. Furthermore, when GAIA distances are available, the spectral energy distribution (SED) can be used to constrain the radii of both components, and if the mass ratio is known, the surface gravities can be obtained with high precision.

\subsection{GSSP: precision}

To check the results of the GSSP code for our systems, we used it to analyse three wide sdB+MS binaries: $\mathrm{BD}+34^{\circ} 1543, \mathrm{BD}+29^{\circ} 3070$ and Feige 87 . These systems were observed with the HERMES spectrograph attached to the Mercator telescope, and the complete analysis was published by Vos et al. (2013). The spectral parameters of the cool companions have been determined in three independent ways. Using the vwA code (Bruntt et al. 2002), fitting synthetic binary spectra to the photometric SED and fitting low-resolution spectra using the XTGRID code (Németh et al. 2012).

The vwa package fits abundances in a semi-automatic way. It first selects the least blended lines in the spectra, and determines the abundances from these lines by calculating synthetic spectra for each line while iteratively changing the input abundance until the equivalent widths (EW) of the observed and synthetic spectrum match. The input parameters $\left(T_{\text {eff }}, \log g\right.$ and $\left.v_{\text {micro }}\right)$ are then manually changed until there is no longer any correlation between iron abundance and EW and excitation potential, and the Fe I and $\mathrm{Fe}$ II abundances match. The rotational velocity is determined by eye. The disadvantage of this method is that the fit can reach a local minimum, and that the method is laborious when many stars need to be analysed. Also, vwA does not allow for the fitting of a dilution factor, and would thus require several assumptions on the spectral parameters of the sdB star to disentangle the spectra. A detailed description of the vwA package can be found in Bruntt et al. (2004, 2010), Bruntt, De Cat \& Aerts (2008) and Bruntt (2009). As VwA does not support the analysis of composite system, the HERMES spectra were first disentangled before a master spectrum was created, which was then used as input for vwA. A detailed description of the analysis method is given in Vos et al. (2013).

The second set of parameters is derived using the SED fitting method. This method fits synthetic SEDs integrated from model atmospheres to photometric SEDs to determine the effective temperature and surface gravity of both components in the binary. The metallicity of the model atmospheres is solar, and $v_{\text {micro }}$ and $v_{\mathrm{r}} \sin i$ cannot be determined in this way. The dilution factor given in Table 4 is the average dilution in the wavelength range 5910$6510 \AA$ A . A detailed description of this method is given in Degroote et al. (2011) and Vos et al. (2012, 2013).

The third set of parameters is derived using XTGRID on fluxcalibrated spectra obtained using the B\&C spectrograph attached to the University of Arizona's 2.3-m Bok telescope located at Kitt Peak. These spectra have a $9-\AA$ resolution. XTGRID fits both the $\mathrm{sdB}$ and cool companion in the system. $v_{\text {micro }}$ and $v_{\mathrm{r}} \sin i$ cannot be determined using the low-resolution data. A detailed explanation of the XTGRID fit is given in Section 4.5. 
For the GSSP analysis, the same method as for the UVES systems was followed exactly. For each of the three systems, a master spectrum is created by shifting all HERMES spectra to the zero-point of the cool companion. The same wavelength ranges of 5910-6270 and $6330-6510 \AA$ are then normalized by hand and used as input for GSSP.

A comparison of the spectral parameters determined by these four methods is given in Table 4. For almost all of the parameters, the GSSP results are in excellent agreement with the results of the other three methods. The only exception is the effective temperature determined by vwA of Feige 87 . As the GSSP results do match well with the results from the SED fit and the XTGRID fit, it is likely that the effective temperature determined by vwA is wrong. This could have happened as a result of the vwa fit being stuck in a local minimum.

This comparison of the GSSP results with the parameters determined using three other independent methods shows that the GSSP code can derive reliable spectral parameters for the cool companion stars in hot subdwarf binaries.

\subsection{XTGRID}

The preceding sections have demonstrated that F- and G-type MS companions of sdB stars can be easily resolved from optical spectra as they have a similar flux contribution and a rich, distinct spectrum. This allows one to disentangle the composite spectra of such binaries from a single observation without knowing the radii or fluxes of the components. Such a binary decomposition is implemented in the steepest-descent chi-squared minimizing spectral fitting algorithm XTGRID, which was first used to estimate the atmospheric parameters of the components in 29 composite spectra binaries from low-resolution data by Németh et al. (2012). XTGRID employs the non-LTE model atmosphere code TLUSTY (Hubeny \& Lanz 1995) for the hot subdwarf component, and interpolated observed templates or theoretical synthetic spectra for the MS companion. Such a decomposition task is relatively easy with low-resolution, high $\mathrm{S} / \mathrm{N}$ flux-calibrated spectra. The binary spectrum can be fitted with the linear combination of the spectra of the two stellar components. In the case of echelle spectroscopy, such as the UVES sample presented here, an absolute flux calibration or continuum normalization is difficult to achieve. Therefore, we applied an improved version of XTGRID to the observations for the highest $\mathrm{S} / \mathrm{N}$ spectrum of each binary system.

XTGRID applies a global optimization in the full parameter space. It fits $T_{\text {eff }}, \log g$, the individual abundances of selected elements and the projected rotation velocity for the sdB star. For the companion, it fits $T_{\text {eff }}, \log g$, metallicity, alpha enhancement and projected rotation velocity. The two models are connected with a wavelengthdependent dilution factor to render composite spectra from the individual stellar components. A RV correction is included in the procedure and the component velocities are determined iteratively along with the surface parameters. The procedure does not check the luminosity ratio of the components, but it seeks for the best possible reproduction of the observations by means of a global chi-squared minimization. In fact, in the case of HE 0505-2806, we found an inconsistent luminosity ratio by assuming the same distance to the components. This suggests that the system is not a physical binary and the companion is a foreground star, confirming the earlier results found by Németh et al. (2012).

Our models for the hot subdwarf star included opacities of $\mathrm{H}$, $\mathrm{He}, \mathrm{C}, \mathrm{N}, \mathrm{O}, \mathrm{Si}$ and $\mathrm{Fe}$ in both the non-LTE model atmosphere and the synthetic spectrum calculation. $\mathrm{Mg}$ was included only in the synthetic spectrum calculation in LTE conditions. The atmospheres were sampled in 50 depth points and converged to a relative change of 0.1 per cent.

We used the synthetic spectral library calculated with the PHOENIX model atmosphere code (version 1; Husser et al. 2013) for the cool companions. The PHOENIX grid covers both the entire parameter range we are interested in and the spectral range of UVES at high resolution including the $\mathrm{Ca}$ II near-infrared triplet. The grid also includes a microturbulent velocity, which is parametrized with the stellar properties. We created a subgrid from scaled solar metallicity models (no alpha enhancement) and limited the grid to $T_{\text {eff }}=4000$ $8000 \mathrm{~K}, \log g=3-5 \mathrm{dex}(\mathrm{cgs})$ and $[\mathrm{Fe} / \mathrm{H}]>-2$ dex. In order to derive the binary parameters and cope with parameter correlations, we found it necessary to fit the entire UVES range simultaneously. The BLUE arm constrains the hot subdwarf properties, while the REDL and REDU arms constrain the companion atmospheric parameters as well as the RVs and the dilution factor. Such a global approach is also preferable to cope with the strong parameter correlations that exist among metallicity, dilution and rotation.

XTGRID starts out from an input model for the binary and improves the fit by successive approximations. To avoid becoming trapped in local minima, XTGRID increases the parameter step sizes (the size of the simplex) regularly and restarts its descent. The error calculation assumes that the steepest descent procedure reduces the correlations for the subdwarf and binary parameters to a minimum and therefore they can be treated separately. According to this, the derived error bars are one-dimensional errors based on the $\chi^{2}$ statistics and neglecting parameter correlations. For the cool companions, we used the $\chi^{2}$ distribution obtained during the last decomposition iteration to derive error bars.

Although the iterative decomposition method employed by XTGRID is more laborious and time-consuming than the GSSP approach, a strong motivation to pursue in this direction was that XTGRID can provide the atmospheric parameters for both components as well as their entire optical spectrum. This allows an improvement of the RV determination for the hot subdwarf and refines the binary parameters further.

\section{ATMOSPHERIC PARAMETERS}

Table 5 gives the spectral parameters derived using GSSP for the companions, together with the upper and lower $1 \sigma$ error values. For most systems, only a lower error on $\log g$ could be given as the fit reached the maximum value. Based on these results, we have a broad range of MS companions with spectral types from $\mathrm{K} 4 \mathrm{~V}$ to F5V. As expected, companions with a higher effective temperature contribute stronger to the composite spectra, which can be seen in the higher dilution factors. The rotational velocity ranges from a slowly rotating $6 \mathrm{~km} \mathrm{~s}^{-1}$, to a very fast rotator with $v_{\mathrm{r}} \sin i=$ $90 \mathrm{~km} \mathrm{~s}^{-1}$. For this small sample, there does not seem to be any correlation between the rotational velocity and the spectral type. The cool companions show a spread in metallicities varying from the very metal-poor MCT $0146-2651$ with $[\mathrm{Fe} / \mathrm{H}]=-0.80$, to two systems, BPS CS 22890-74 and J 03582-3609, with a super solar metallicity. The best fitting GSSP models are shown in Fig 4.

The $\mathrm{sdB}$ components have a complicated formation history including diffusion processes that can cause chemical peculiarities (Heber 1998). This makes it difficult, if not impossible, to estimate the age of the sdBs from atmospheric parameters. When there has been little to no mass transfer in the history of the binary, the metallicity of the cool companion can be used as an age indicator. The 
Table 5. Spectroscopic parameters for nine cool companions of the sdB binaries observed with the UVES. The parameters determined using the GSSP code are given on the first row, while those determined by XTGRID are given on the second row. The errors provided for GSSP are the $1 \sigma$ errors, and those for XTGRID are the 60 per cent confidence intervals. The XTGRID errors do not take parameter correlations into account, while the GSSP errors do. The dilution is the average dilution of the cool companion, $d=F_{\mathrm{MS}} /\left(F_{\mathrm{MS}}+F_{\mathrm{sdB}}\right)$, in the wavelength range 5900-6500 Å. See also Section 5.

\begin{tabular}{|c|c|c|c|c|c|}
\hline Name & $\begin{array}{l}T_{\text {eff }} \\
(\mathrm{K})\end{array}$ & $\begin{array}{l}\log g \\
(\operatorname{dex})\end{array}$ & $\begin{array}{c}{[\mathrm{Fe} / \mathrm{H}]} \\
(\mathrm{dex})\end{array}$ & $\begin{array}{c}v_{\text {rot }} \sin i \\
\left(\mathrm{~km} \mathrm{~s}^{-1}\right)\end{array}$ & Dilution \\
\hline BPS CS 22890-74 & $\begin{array}{c}5630_{-220}^{+170} \\
5650_{-70}^{+70}\end{array}$ & $\begin{array}{l}4.50_{-0.20}^{+0.00} \\
4.72_{-0.26}^{+0.23}\end{array}$ & $\begin{array}{l}0.10_{-0.10}^{+0.20} \\
0.50_{-0.10}^{+0.10}\end{array}$ & $\begin{array}{c}7_{-1}^{+1} \\
9_{-0.3}^{+0.3}\end{array}$ & $\begin{array}{l}0.45_{-0.05}^{+0.05} \\
0.42_{-0.01}^{+0.01}\end{array}$ \\
\hline HE 0430-2457 & $\begin{array}{r}4620_{-200}^{+170} \\
4790_{-250}^{+250}\end{array}$ & $\begin{array}{l}4.50_{-0.50}^{+0.00} \\
5.00_{-0.20}^{+0.40}\end{array}$ & $\begin{array}{r}-0.35_{-0.25}^{+0.30} \\
-0.50_{-0.30}^{+0.10}\end{array}$ & $\begin{array}{l}30_{-3}^{+3} \\
36_{-5}^{+5}\end{array}$ & $\begin{array}{l}0.20_{-0.05}^{+0.05} \\
0.24_{-0.01}^{+0.01}\end{array}$ \\
\hline J 02286-3625 & $\begin{array}{l}5330 \\
5090_{-90}^{+180} \\
+150\end{array}$ & $\begin{array}{l}4.50_{-0.50}^{+0.00} \\
3.80_{-0.20}^{+0.20}\end{array}$ & $\begin{array}{l}-0.15_{-0.15}^{+0.10} \\
-0.32_{-0.10}^{+0.10}\end{array}$ & $\begin{array}{c}90_{-3}^{+5} \\
99_{-10}^{+8}\end{array}$ & $\begin{array}{l}0.30_{-0.05}^{+0.05} \\
0.40_{-0.01}^{+0.01}\end{array}$ \\
\hline J 03154-5934 & $\begin{array}{r}6080_{-190}^{+190} \\
6070_{-100}^{+150}\end{array}$ & $\begin{array}{l}4.40_{-0.35}^{+0.10} \\
4.34_{-0.10}^{+0.10}\end{array}$ & $\begin{array}{l}-0.30_{-0.10}^{+0.15} \\
-0.03_{-0.10}^{+0.20}\end{array}$ & $\begin{array}{c}23_{-1}^{+1} \\
24_{-0.5}^{+0.3}\end{array}$ & $\begin{array}{l}0.55_{-0.05}^{+0.05} \\
0.55_{-0.02}^{+0.02}\end{array}$ \\
\hline J 03582-3609 & $\begin{array}{l}6030+220 \\
6200_{-240}^{+110}\end{array}$ & $\begin{array}{l}4.20_{-0.40}^{+0.30} \\
4.50_{-0.07}^{+0.10}\end{array}$ & $\begin{array}{l}0.00_{-0.15}^{+0.15} \\
0.50_{-0.30}^{+0.30}\end{array}$ & $\begin{array}{c}30_{-2}^{+2} \\
31_{-1.2}^{+1.2}\end{array}$ & $\begin{array}{l}0.75_{-0.10}^{+0.10} \\
0.76_{-0.01}^{+0.01}\end{array}$ \\
\hline JL 277 & $\begin{array}{c}6550_{-200}^{+200} \\
6270_{-130}^{+80}\end{array}$ & $\begin{array}{r}4.50_{-0.50}^{+0.00} \\
4.11_{-0.15}^{+0.15}\end{array}$ & $\begin{array}{l}-0.40_{-0.15}^{+0.15} \\
-0.50_{-0.10}^{+0.10}\end{array}$ & $\begin{array}{c}33_{-2}^{+2} \\
32_{-1.3}^{+2.3}\end{array}$ & $\begin{array}{l}0.60_{-0.10}^{+0.05} \\
0.62_{-0.01}^{+0.01}\end{array}$ \\
\hline MCT 0146-2651 & $\begin{array}{l}5910_{-220}^{+160} \\
5800_{-100}^{+100}\end{array}$ & $\begin{array}{l}4.50_{-0.15}^{+0.00} \\
4.50_{-0.30}^{+0.20}\end{array}$ & $\begin{array}{l}-0.80_{-0.10}^{+0.00} \\
-1.00_{-0.10}^{+0.10}\end{array}$ & $\begin{array}{c}6_{-2}^{+2} \\
5.6_{-0.6}^{+0.8}\end{array}$ & $\begin{array}{l}0.40_{-0.05}^{+0.05} \\
0.42_{-0.01}^{+0.01}\end{array}$ \\
\hline PB 6148 & $\begin{array}{c}6540_{-120}^{+160} \\
6250_{-50}^{+50}\end{array}$ & $\begin{array}{l}4.50_{-0.50}^{+0.00} \\
3.73_{-0.10}^{+0.07}\end{array}$ & $\begin{array}{l}-0.35_{-0.10}^{+0.05} \\
-0.46_{-0.10}^{+0.10}\end{array}$ & $\begin{array}{c}15_{-1}^{+1} \\
15_{-0.4}^{+0.4}\end{array}$ & $\begin{array}{c}0.94_{-0.03}^{+0.03} \\
1.00_{-0.00}^{+0.01}\end{array}$ \\
\hline PG 1514+034 & $\begin{array}{l}5630_{-350}^{+270} \\
5900_{-185}^{+130}\end{array}$ & $\begin{array}{c}4.50_{-0.50}^{+0.00} \\
5.00_{-0.10}^{+0.25}\end{array}$ & $\begin{array}{c}-0.50_{-0.15}^{+0.30} \\
0.00_{-0.10}^{+0.10}\end{array}$ & $\begin{array}{c}18_{-2}^{+2} \\
20_{-0.8}^{+0.7}\end{array}$ & $\begin{array}{l}0.44_{-0.10}^{+0.10} \\
0.32_{-0.01}^{+0.01}\end{array}$ \\
\hline
\end{tabular}

spread in metallicity that we observe here suggests a wide range of ages for these systems.

The XTGRID results for the companion stars are given on the second row for each system in Table 5. The effective temperature and surface gravity of the $\mathrm{sdB}$ components determined using XTGRID are given in Table 6. The abundances of $\mathrm{He}, \mathrm{C}, \mathrm{N}, \mathrm{O}, \mathrm{Mg}, \mathrm{Si}$ and Fe for the sdB component are given in Table 7. PB 6148 is missing in the latter two tables as it does not have an sdB component (see Section 5.3). A section of the total fitted region with XTGRID for each system is shown in Figs 5 and 6. These figures show the bestfitting binary spectra, as well as the contribution of the $\mathrm{sdB}$ and the cool companion.

When comparing the XTGRID results with the GSSP results, we have to take into account the fact that the errors given for the XTGRID parameters do not include the correlations between the parameters and, as such, are smaller than the GSSP errors. For the nine systems discussed here, the atmospheric parameters determined with the two methods are in good agreement. The largest difference between the two methods is the derived metallicity. For about half of the systems, the XTGRID results find a significantly higher metallicity. This difference is a result of the different model atmospheres employed by the two methods, which is discussed in the next section.

\section{1 sdB He sequence}

XTGRID was originally designed to fit single-lined hot subdwarf spectra, and binary decomposition was added later to be able to fit the considerable number of hot subdwarfs in composite spectrum binaries. The high resolution of the UVES and the adequate S/N allows us to separate the components and to derive precise atmospheric parameters for both the subdwarf and the cool companion. Such a task is usually not possible using low-resolution data.

Edelmann et al. (2003) found a positive correlation between $T_{\text {eff }}$ and $\log (n \mathrm{He} / n \mathrm{H})$ of hot subdwarfs and that two such He sequences exist. We found that all subdwarfs in the UVES sample follow this trend and clearly belong to the He-rich sequence. Past investigations revealed that $\mathrm{sdB}$ stars along the He-rich sequence appear in two major clumps and these clumps correlate with the binary and pulsation properties of the stars. Németh et al. (2012) found that most hot subdwarfs in composite spectra in the GALEX sample belong to the higher temperature, gravity and helium abundance group. Fig. 7 shows that all sdB binaries observed with the UVES can be associated with both of these clumps; however, the current sample is too small to draw any conclusion. Our efforts in collecting a larger sample will reveal the relation between the EHB morphology and composite spectrum binaries.

The surface gravity of the two sdOB systems (MCT 0146-2651 and PG 1514+034) suggests that these subdwarfs have started evolving off the extreme horizontal branch (EHB) while their helium abundance is still consistent with EHB stars.

The spectral analysis provided reliable measurements for $T_{\text {eff }}$, $\log g$ and the He abundance, as listed in Table 6. Therefore, the fits to binary systems have more free parameters; even though the lines of $\mathrm{Mg}$ and $\mathrm{Si}$ are clearly present in the spectra, we can report only upper limits of their abundances in Table 7 at the given level of confidence. Despite the fact that metal abundances are difficult to measure from optical spectra, the abundances we found are comparable to values in the literature (O'Toole \& Heber 2006; Blanchette et al. 2008). 


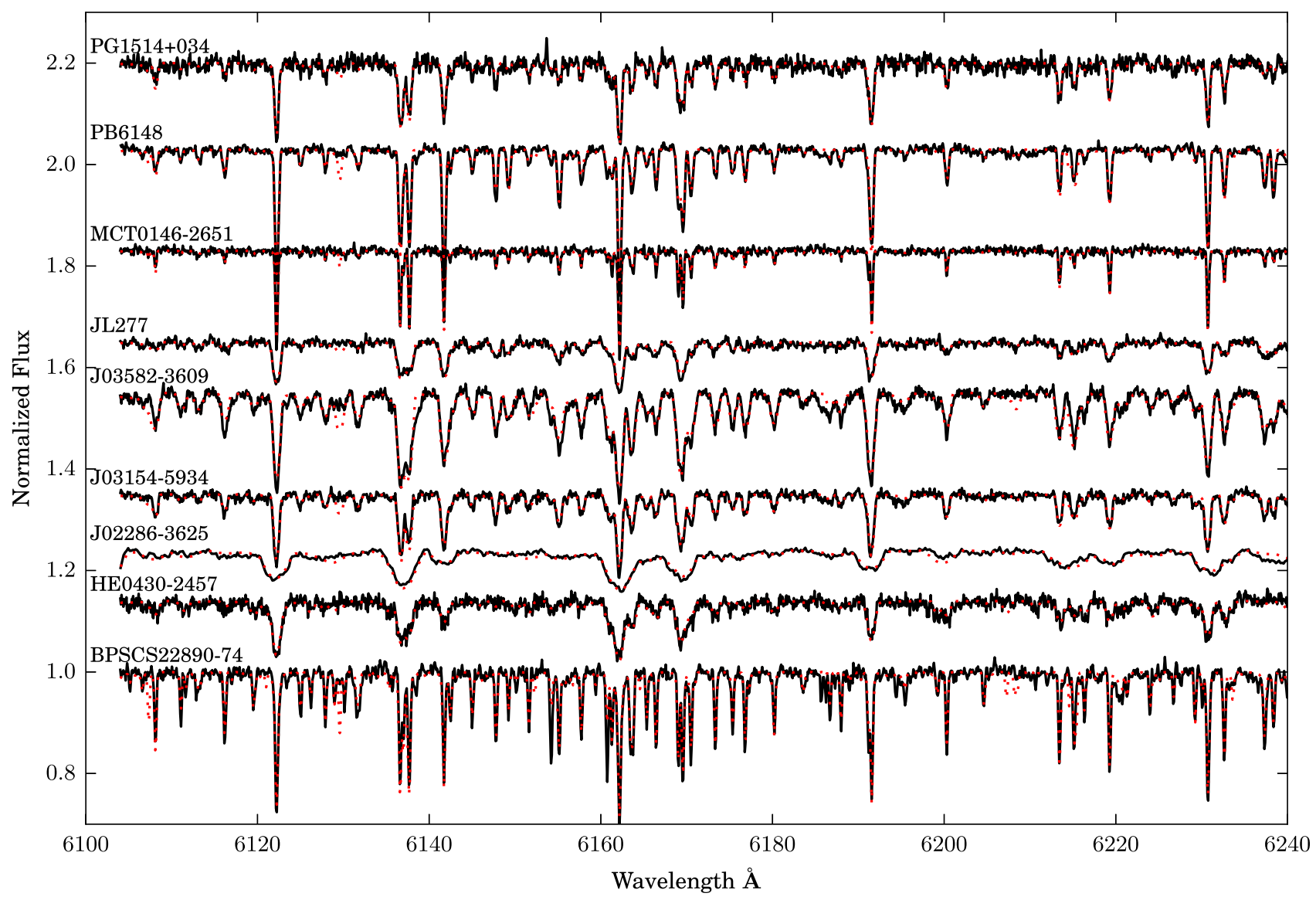

Figure 4. Observed normalized spectra (black full line) and best-fitting GSSP model (red dotted line) for a section of the wavelength range used to determine the spectroscopic parameters with GSSP.

Table 6. $T_{\text {eff }}$ and $\log g$ of the sdB components derived using the XTGRID code. The upper and lower errors are the 60 per cent confidence intervals. PB 6148 is not included in this table as it does not contain a hot subdwarf (see Section 5).

\begin{tabular}{lcc}
\hline Name & $\begin{array}{c}T_{\text {eff }} \text { sdB } \\
(\mathrm{K})\end{array}$ & $\begin{array}{c}\log g \text { sdB } \\
(\text { dex })\end{array}$ \\
\hline BPS CS 22890-74 & $22890_{-320}^{+1320}$ & $5.24_{-0.07}^{+0.18}$ \\
HE 0430-2457 & $26500_{-600}^{+500}$ & $5.38_{-0.10}^{+0.10}$ \\
J 02286-3625 & $28790_{-440}^{+430}$ & $5.38_{-0.08}^{+0.08}$ \\
J 03154-5934 & $26740_{-780}^{+1000}$ & $5.39_{-0.14}^{+0.13}$ \\
J 03582-3609 & $31330_{-1130}^{+2530}$ & $5.80_{-0.60}^{+0.21}$ \\
JL 277 & $25410_{-940}^{+320}$ & $5.44_{-0.23}^{+0.04}$ \\
MCT 0146-2651 & $36460_{-610}^{+530}$ & $5.72_{-0.08}^{+0.07}$ \\
PG 1514+034 & $34590_{-800}^{+750}$ & $5.53_{-0.13}^{+0.14}$ \\
\hline
\end{tabular}

Metals are at subsolar abundances in all stars. Carbon and oxygen show a large scatter from star to star, while nitrogen is the most abundant light metal. Iron is very close to the solar iron abundance in agreement with the results of Geier (2013).

\subsection{Metallicity difference between the LLMODELS and PHOENIX models}

Although the atmospheric parameters of the cool companion determined with GSSP and XTGRID correspond well overall, there is a discrepancy in the metallicity for about half of the systems. For BPS CS 22890-74, HE 0430-2457, J 03154-5934, J 03582-3609 and PG 1514+034, the metallicity determined with XTGRID is significantly higher than the GSSP metallicity. The difference is roughly 0.4 dex. The reason for this offset might be related to the different models used for the cool companion.

GSSP uses Line by Line opacity models (LLMODELS; Shulyak et al. 2004), which are LTE plane-parallel stellar model atmospheres for early- and intermediate-type stars using the Vienna Atomic Line Database (VALD; Ryabchikova et al. 2015). In the current analysis, we used XTGRID with PHOENIX models (Husser et al. 2013), which also assume LTE but the modelling geometry, atomic data input and numeric algorithms are likely to be different from LLMODELS. Fig. 8 shows a synthetic spectrum calculated with both the LLMODELS and PHOENIX models. This spectrum has $T_{\text {eff }}=5700 \mathrm{~K}, \log g=4.0 \mathrm{dex}$, $[\mathrm{Fe} / \mathrm{H}]=0.0,[\alpha / \mathrm{Fe}]=0.0$, and is convolved to fit the resolution of our UVES spectra. In the wavelength range shown, it is clear that there is a significant difference in some of the Fe lines between the two models, with some lines being clearly present in one model but totally absent in the other. Other lines, such as Ca I, are exactly the same in both models. With this in mind, we attribute the different metallicities reported by both fitting algorithms to the differences in the synthetic atmospheres they use

Even though the effective temperature, surface gravity, rotation and dilution derived for the nine systems given in Table 5 are comparable between the LLMODELS and PHOENIX models, the metallicity is not. This comparison shows that the atmosphere models used to determine atmospheric parameters have an influence on those 
Table 7. Surface abundances for the sdB components determined with XTGRID. The errors are the 60 per cent confidence intervals. PB 6148 is not included in this table as it does not contain a hot subdwarf (see Section 5).

\begin{tabular}{|c|c|c|c|c|c|c|c|}
\hline \multirow[t]{2}{*}{ Name } & \multicolumn{6}{|c|}{$\log (n X / n \mathrm{H}) \pm \log (1+\delta n X / n X)$} & \multirow[b]{2}{*}{$\mathrm{Fe}$} \\
\hline & $\mathrm{He}$ & $\mathrm{C}$ & $\mathrm{N}$ & $\mathrm{O}$ & $\mathrm{Mg}$ & $\mathrm{Si}$ & \\
\hline BPS CS 22890-74 & $-3.07_{-0.36}^{+0.29}$ & $<-4.34$ & $<-3.70$ & $<-3.65$ & $<-4.62$ & $<-5.22$ & $-4.54_{-2.93}^{+0.75}$ \\
\hline HE 0430-2457 & $\begin{array}{r}-0.50 \\
-2.59_{-0.12}^{+0.19}\end{array}$ & $-4.06_{-0.45}^{+0.20}$ & $-4.38_{-0.80}^{+0.36}$ & $-4.31_{-0.68}^{+0.30}$ & $<-4.25$ & $-4.52_{-0.48}^{+0.48}$ & $<-4.50$ \\
\hline J 02286-3625 & $-2.55_{-0.16}^{+0.18}$ & $\begin{array}{r}-0.45 \\
-4.70_{-0.68}^{+0.54}\end{array}$ & $-4.44_{-0.31}^{+0.00}$ & $\begin{array}{r}-4.53_{-0.34}^{+0.16} \\
-0.00\end{array}$ & $<-5.18$ & $<-6.25$ & $<-4.77$ \\
\hline J 03154-5934 & $-2.80_{-0.23}^{+0.41}$ & $<-3.96$ & $-4.64_{-1.00}^{+0.54}$ & $-3.81_{-0.61}^{+0.18}$ & $<-4.64$ & $<-5.66$ & $<-4.31$ \\
\hline J 03582-3609 & $-1.23_{-0.67}^{+0.40}$ & $-3.24_{-1.66}^{+0.72}$ & $<-3.09$ & $-3.02_{-0.59}^{+0.01}$ & $<-2.02$ & $-3.86_{-1.04}^{+0.46}$ & $<-3.47$ \\
\hline JL 277 & $-2.23_{-0.20}^{+0.09}$ & $-4.59_{-0.72}^{+0.40}$ & $-4.47_{-0.61}^{+0.27}$ & $-3.65_{-0.42}^{+0.15}$ & $<-4.97$ & $<-5.34$ & $<-4.27$ \\
\hline МCT 0146-2651 & $-1.57_{-0.10}^{+0.13}$ & $<-5.24$ & $<-4.37$ & $<-4.65$ & $<-4.34$ & $<-5.38$ & $-4.36_{-0.49}^{+0.53}$ \\
\hline PG 1514+034 & $-1.90_{-0.17}^{+0.18}$ & $<-4.79$ & $-4.74_{-1.11}^{+0.99}$ & $<-4.61$ & $<-4.40$ & $<-5.41$ & $-5.24_{-2.66}^{+1.54}$ \\
\hline
\end{tabular}

parameters, and that if a comparison of the atmospheric parameters of different stars is made, it should ideally be done using the same atmosphere models.

This metallicity difference does not show up when comparing the XTGRID results for the three test systems in Section 4.4. This is because those systems were analysed using an earlier version of XTGRID with ATLAS models (Bertone et al. 2004) in the range of $3700-7200 \AA$, instead of the PHOENIX models used in the analysis of the UVES systems.

\subsection{PB 6148}

PB 6148 was identified by Berger \& Fringant (1980) as an sdOB binary with an F-type companion based on low-resolution spectroscopy (dispersion $\sim 80 \AA \mathrm{mm}^{-1}$ ) with the ' $\mathrm{D}$ ' spectrograph at the $80-\mathrm{cm}$ telescope at Observatoire de Haute-Provence. However, in our high-resolution UVES observations, there is no visible spectral signature of an $\mathrm{sdB}$ component. Even though there is no indication of an sdB component in the spectra, the spectral fit of the F-type star with GSSP indicates that there is a component present, contributing about 5 per cent of the total light, while the XTGRID fit attributes 10 per cent of the total light to the unidentified companion.

In total, three UVES spectra of PB 6148 were obtained on HJD 245 5908, 2455934 and 245 6851. For these spectra, the RVs of the F-type star were determined using a cross-correlation with a template spectra, as explained in Section 4.1. The F-type star shows RV variations with a maximum amplitude of $2.5 \mathrm{~km} \mathrm{~s}^{-1}$. This variation is five times larger than the uncertainty on the RV measurements, indicating that PB 6148 is likely a binary system. There is also a very weak line visible at $\lambda 5875 \AA$, which might be a He I line. RV measurements of this line alone show a movement in antiphase with that of the F-type star, but the errors are of the same order as the amplitude. Based on the available data, we cannot identify the secondary component of PB 6148.

\section{MASS ACCRETION BY THE COMPANIONS}

The evolutionary state of some companions to sdB stars has been used as an argument that the companions could not have accreted much mass during the RLOF phase. If the companion star is slightly evolved, then it cannot have accreted much matter, as a star with a lower initial mass would not have had enough time to evolve (see, e.g. Vos et al. 2012). However, no attempt has been made to quantify the amount of accreted mass.

By measuring the current rotational velocity and comparing this to the initial synchronized state, an estimate of the accreted mass can be made based on the amount of angular momentum that needs to be transferred on to the companion to reach the observed rotation velocity. The angular momentum of the companion based on the rotational velocity is given by

$\Omega=r_{\mathrm{g}} M_{\mathrm{c}} R_{\mathrm{c}}^{2} \omega$,

where $r_{\mathrm{g}}$ is the gyration radius of the companion, $M_{\mathrm{c}}$ and $R_{\mathrm{c}}$ are the mass and radius of the companion, respectively, and $\omega$ is the angular velocity, which can be calculated as $\omega=v_{\text {rot }} / R=2 \pi / P_{\text {spin }}$, where $P_{\text {spin }}$ is the spin period. The change in angular momentum before and after mass transfer is then

$\triangle \Omega=r_{\mathrm{g}} M_{\mathrm{c}} R_{\mathrm{c}}^{2}\left(\frac{v_{\text {rot } \mathrm{f}}}{R_{\mathrm{c}}}-\frac{2 \pi}{P_{\text {spin }, \mathrm{i}}}\right)$,

where $P_{\text {spin, } \mathrm{i}}$ is the initial spin period of the companion before the onset of RLOF and $v_{\text {rot, f }}$ is the observed rotational velocity. As the binary would have been circularized and synchronized before the onset of RLOF, the initial spin period equals the orbital period before RLOF $P_{\text {spin,i }}=P_{\text {orb,i. }}$. The orbital period before RLOF is estimated between 500 and $900 \mathrm{~d}$, as this corresponds to the separation necessary to initiate RLOF near the tip of the RGB (Vos et al. 2015)

Equation (2) assumes that the radius of the companion does not change significantly between the onset of RLOF, and the moment when the system is observed. The time-scales involved here are short compared to the MS lifetime of the companion, so this assumption is valid in the case where the amount of mass accreted by the companion is low.

\subsection{Angular momentum transfer during RLOF}

The amount of angular momentum transfer differs depending on whether the mass flow directly hits the companion (ballistic mass transfer), or first forms an accretion disc and is only then accreted on to the companion (Keplerian disc mass transfer). To determine which regime is applicable in this case, we calculate the minimum distance of the mass stream to the companion following the fitting formula from Ulrich \& Burger (1976) based on Lubow \& Shu (1975):

$R_{\min }=0.0425 a\left(q+q^{2}\right)^{1 / 4}$.

Here, $a$ is the separation and $q=M_{\mathrm{c}} / M_{\mathrm{sdB}}$. If the distance between the mass stream and the centre of mass of the companion is larger than its radius, an accretion disc will be formed. In the case of wide sdB binaries, we calculate the minimum distance to the companion for systems with $P_{\mathrm{i}}=500-900 \mathrm{~d}, P_{\mathrm{f}}=700-1300 \mathrm{~d}$, an sdB progenitor mass between 1.5 and $2.0 \mathrm{M}_{\odot}$, an sdB mass of $0.47 \mathrm{M}_{\odot}$, and 


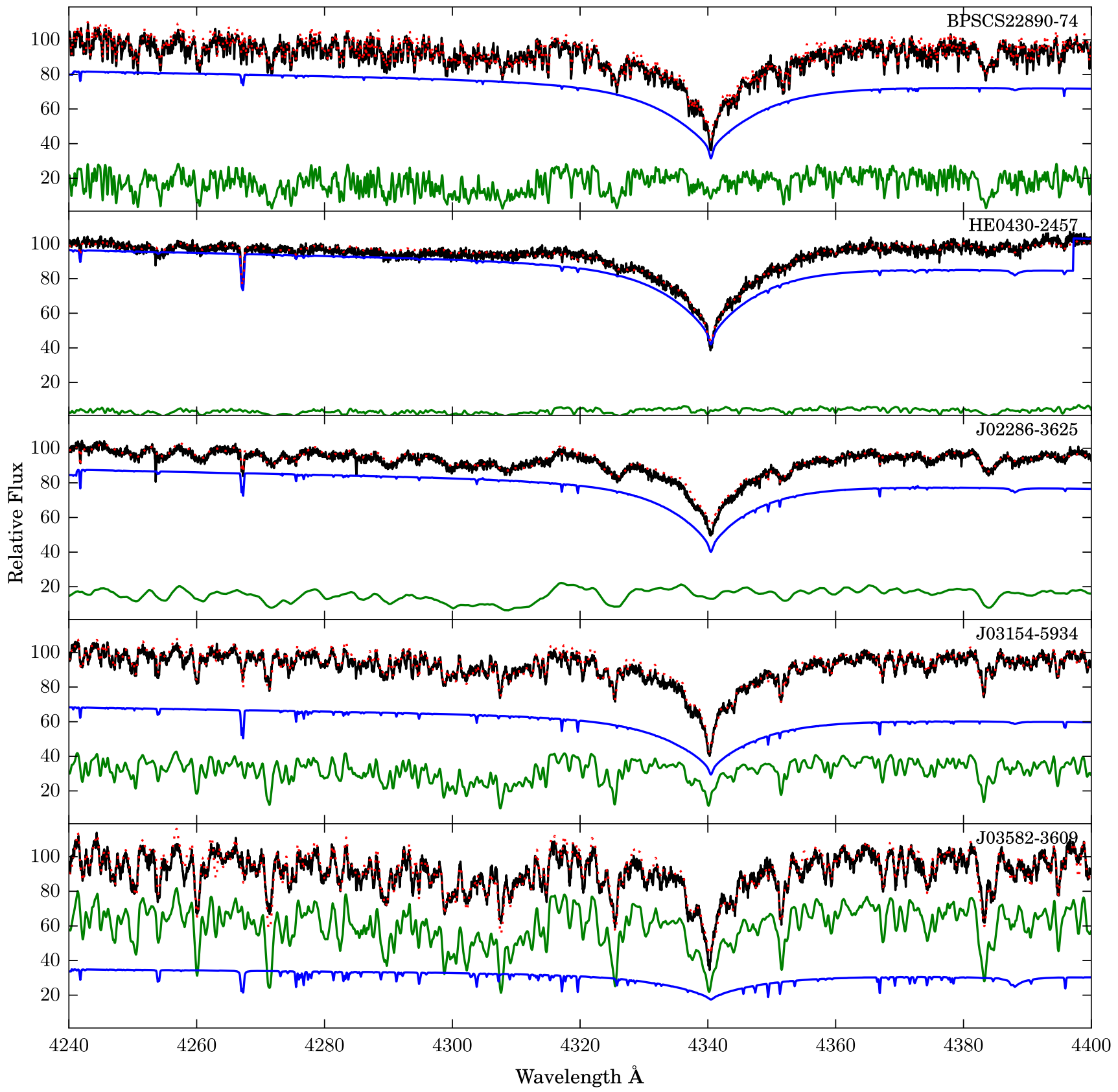

Figure 5. Observed UVES spectra (black full line) and best-fitting XTGRID model. The cool companion contribution is shown as a green line, the sdB contribution is shown as a blue line and the binary model is shown as a red dotted line. The flux is scaled so that the average continuum flux of the observed spectrum equals 100 for easy comparison between the systems. Interactive figures are available at http://astroserver.org/KW32YZ/.

a companion with a mass of $1 \mathrm{M}_{\odot}$. For these parameters, the minimum distance between the mass stream and the companion varies between 15 and $33 \mathrm{R} \odot$. This is in all cases much larger than the radius of the companion, and the mass lost from the $\mathrm{sdB}$ progenitor will thus never directly hit the companion.

The angular momentum accreted by the companion from an accretion disc is the angular momentum of the inner radius of the Keplerian accretion disc around the companion, which is taken at the surface of the companion,

$\delta \Omega_{\mathrm{acc}}=\sqrt{G M_{\mathrm{c}} R_{\mathrm{c}}} \delta M_{\mathrm{acc}}$, where $\delta \Omega_{\mathrm{acc}}$ is the accreted angular momentum per unit mass accreted by the companion $\delta M_{\text {acc }}$ and $G$ is the gravitational constant.

\subsection{Angular momentum loss during the sdB phase}

To check if the current observed rotational velocity of the companion is the same as its rotational velocity after the end of RLOF, we compare the sdB lifetime with the synchronization time-scales for the companion. Using the formalism of Zahn (1977), the synchronization time-scale of the companion is given by

$t_{\text {syn }}=\frac{1}{6 q^{2} k_{2}}\left(\frac{M_{\mathrm{c}} R_{\mathrm{c}}}{L_{\mathrm{c}}}\right)^{1 / 3} \frac{I_{\mathrm{c}}}{M_{\mathrm{c}} R_{\mathrm{c}}^{2}}\left(\frac{a}{R_{\mathrm{c}}}\right)^{6}$, 


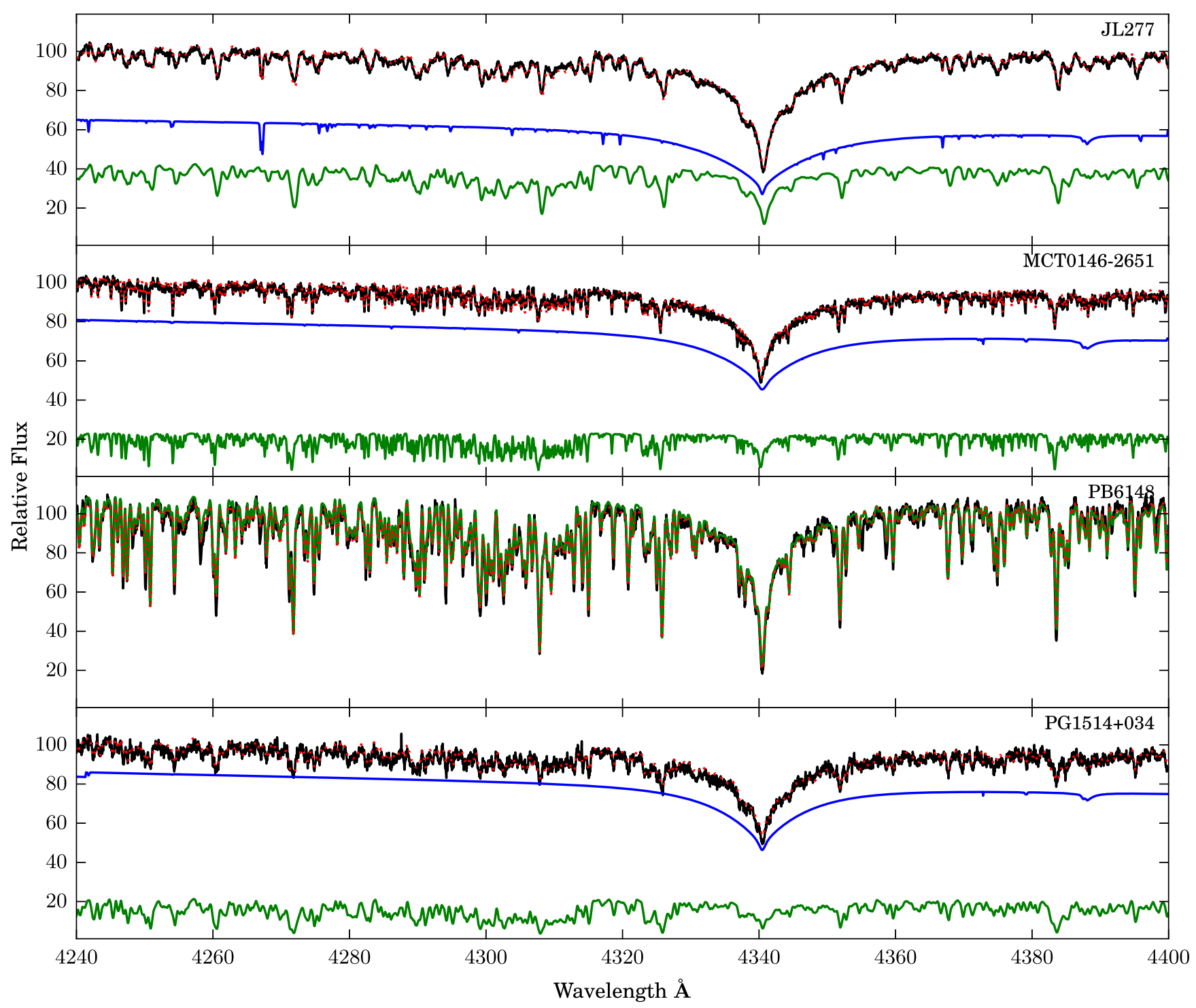

Figure 6. Observed UVES spectra (black full line) and best-fitting XTGRID model. The cool companion contribution is shown as a green line, the sdB contribution is shown as a blue line and the binary model is shown as a red dotted line. The flux is scaled so that the average continuum flux of the observed spectrum equals 100 for easy comparison between the systems. Interactive figures are available at http://astroserver.org/KW32YZ/.

where $q=M_{\mathrm{sdB}} / M_{\mathrm{c}}, k_{2}$ is the apsidal motion constant, $L_{\mathrm{c}}$ is the luminosity of the companion, $I_{\mathrm{c}}=r_{\mathrm{g}} M_{\mathrm{c}} R_{\mathrm{c}}^{2}$ is the moment of inertia and $a$ is the separation. Brooker \& Olle (1955) calculated apsidal motion constants using polytrope models. For an MS star with polytropic index $n=3, k_{2}=0.0144$. Gyration radii were calculated by Claret \& Gimenez (1989), also using polytrope models, which for MS stars results in $r_{g}=0.076$.

Assuming a solar-type companion, and an average separation of $a=500 \mathrm{R}_{\odot}$, the synchronization time-scale exceeds the Hubble time. Synchronization will thus not affect the rotational velocity of the companions after the end of RLOF.

Synchronization is not the only way to lose spin angular momentum. In this derivation, we ignore other angular-momentum-loss effects (e.g. due to stellar winds or magnetic fields), as these effects are difficult to quantify.

\subsection{Accreted mass}

By linking the accreted angular momentum per unit mass (equation 4), to the observed change in angular momentum (equation 2), the amount of mass accreted by the companion can be calculated as

$M_{\mathrm{acc}}=r_{\mathrm{g}} \sqrt{\frac{M_{\mathrm{c}} R_{\mathrm{c}}^{3}}{G}}\left(\frac{v_{\mathrm{rot}, \mathrm{f}}}{R_{\mathrm{c}}}-\frac{2 \pi}{P_{\mathrm{orb}, \mathrm{i}}}\right)$.

As only spectral parameters of the companions are known, we use the average masses and radii based on the spectral types to determine the accreted mass. The spectral types, masses and radii were matched to the observations using the results of Pecaut \& Mamajek (2013) and the empirical mass-radius relation of Demircan \& Kahraman (1991). In deriving the accreted mass, an error of 10 per cent was assumed on the masses and radii.

The inclination of these systems is not known, so we calculated a lower limit on the accreted mass using $i=90^{\circ}$, and an upper limit using $i=21^{\circ}$, which is the lowest inclination measured for a wide sdB binary. Table 8 gives the accreted mass in both cases, together with the spectral type and derived masses and radii for all analysed systems.

From these results, it is clear that there is very little mass accretion on to the companions. The fastest rotator, J 02286-3625, would 

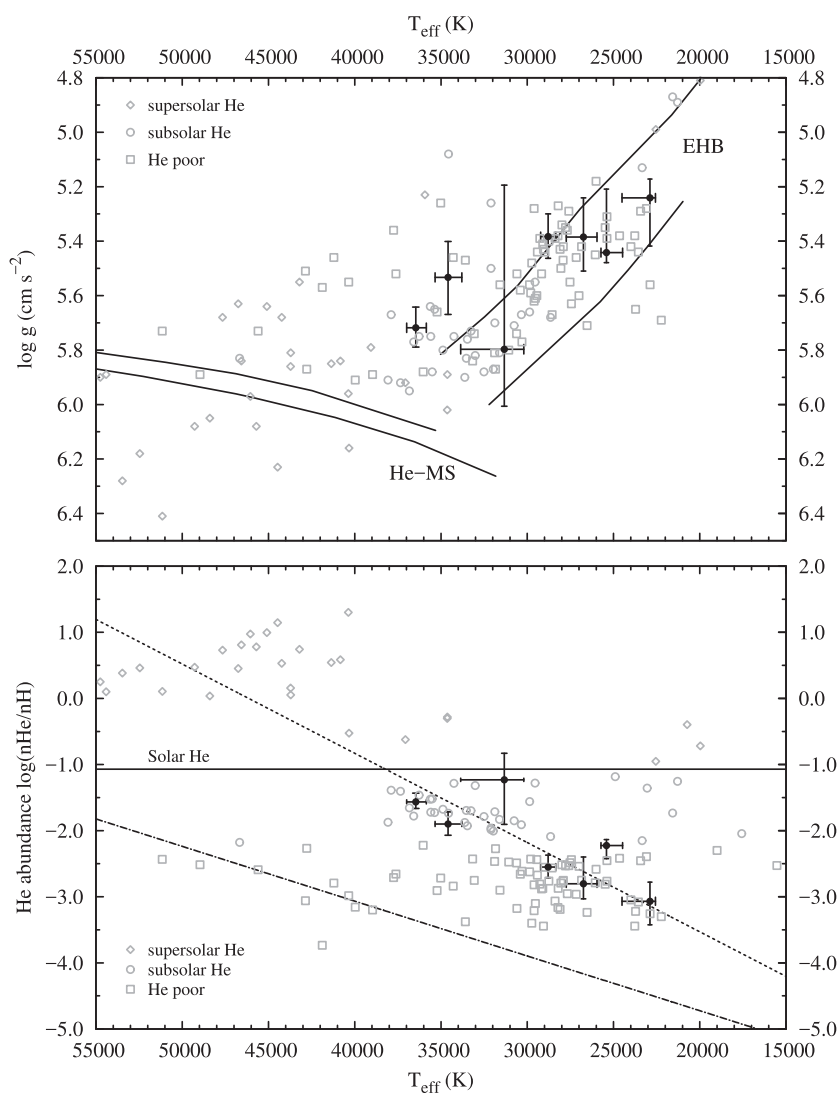

Figure 7. Hot subdwarfs from the UVES sample in the $T_{\text {eff }}-\log g$ (upper panel) and $T_{\text {eff }}-\log (n \mathrm{He} / n \mathrm{H})$ (bottom panel) planes. The grey data points provide a reference for the subdwarf atmospheric properties and are taken from the GALEX sample (Németh et al. 2012). The EHB and He main sequence (He-MS) are marked in the top panel. The horizontal line in the bottom panel shows the solar helium abundance. The dashed line in the $T_{\text {eff }}-$ $\log (n \mathrm{He} / n \mathrm{H})$ panel is the correlation taken from an independent analysis by Edelmann et al. (2003). The dash-dotted line (bottom panel) is the lower boundary of the surface helium abundances found in the GALEX sample. only need to accrete at most $0.04 \mathrm{M}_{\odot}$ in order to reach its observed rotational velocity if this system is observed at an inclination of $21^{\circ}$. On average, these systems have accreted between 0.005 and $0.013 \mathrm{M}_{\odot}$ in the $i=90^{\circ}$ and $i=21^{\circ}$ cases, respectively.

\subsection{Duration of accretion}

The maximum rate at which a star can accrete matter is defined by the Eddington luminosity. If one assumes that the total luminosity of the star originates from energy deposited on to the star by the accreted matter, then the Eddington accretion limit is given by

$\dot{M}_{\text {edd }}=\frac{4 \pi c}{\kappa} R_{\mathrm{c}}$,

where $c$ is the speed of light and $\kappa$ is the opacity that, in the simplest case, can be taken from Thompson scattering $\kappa=0.4 \mathrm{~cm}^{2} \mathrm{~g}^{-1}$. If applied to the accretion masses derived here (Section 6.3), the minimum time necessary to accrete this matter is of the order of years to decades. Table 8 gives the time-scales calculated for the amount of mass accreted when the inclination is $i=21^{\circ}$. This is the minimum duration, and the real time is likely longer, as the accretion takes place from an accretion disc while the Eddington limit is an upper limit derived for spherically symmetric accretion, and the Thompson scattering opacity is not the best approximation for an MS star. However, the time-scales derived here allow the prediction of thermal time-scale mass loss for red giants.

\subsection{Evolutionary considerations}

The determination of the mass accretion by the companion based on its rotational velocity is only valid if there has indeed been a mass-transfer phase in the history of the system. In the case of very wide initial orbits, no mass transfer would take place during the RGB phase, and the measured rotational velocity of the cool companion would be its initial rotational velocity. The upper limit on the initial period to allow for mass transfer during the RGB phase is around $1000 \mathrm{~d}$. Depending on the mass-loss formalism used, these systems can reach orbital periods of $1600 \mathrm{~d}$ or more

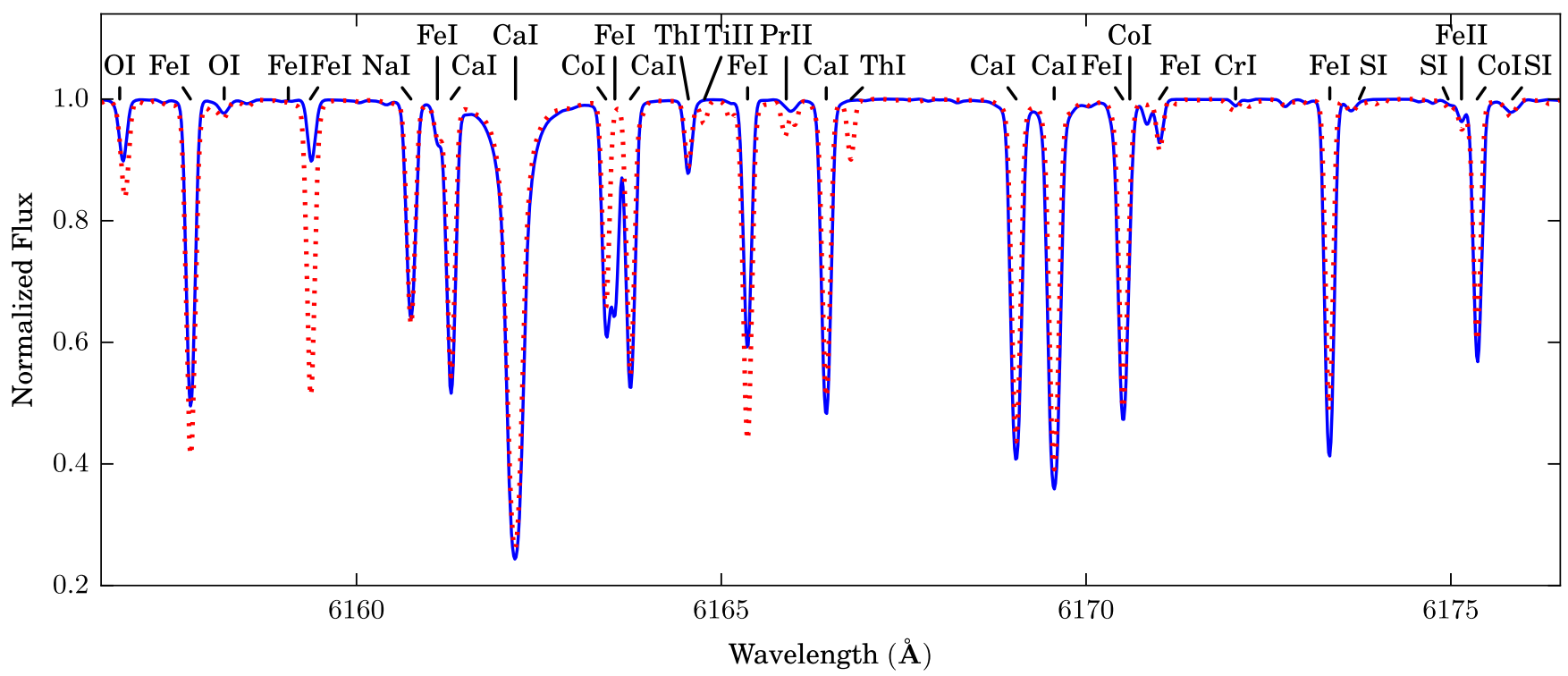

Figure 8. Comparison of an LLmodels synthetic spectrum (blue full line) with a PHOENIX synthetic spectrum (red dotted line). Both spectra are calculated for $T_{\text {eff }}=5700 \mathrm{~K}, \log g=4.0 \mathrm{dex},[\mathrm{Fe} / \mathrm{H}]=0.0,[\alpha / \mathrm{Fe}]=0.0$, and convolved to fit the UVES resolution. The stronger lines are identified above the spectra. There is a clear difference in the strength of several Fe lines visible, with some lines being clearly present in one model but totally absent in the other. 
Table 8. The amount of mass that needs to be accreted on to the companions to reach the observed rotational velocities for two possible inclinations. The masses and radii are based on the spectral type. The minimum duration of mass accretion $\tau_{\min }$ is given for $M_{\mathrm{acc}}\left(i=21^{\circ}\right)$. See Section 6 for details.

\begin{tabular}{lccccccr}
\hline Name & $\begin{array}{c}\text { Spectral } \\
\text { type }\end{array}$ & $\begin{array}{c}\text { Mass } \\
\left(\mathrm{M}_{\odot}\right)\end{array}$ & $\begin{array}{c}\text { Radius } \\
\left(\mathrm{R}_{\odot}\right)\end{array}$ & $\begin{array}{c}v_{\text {rot }} \sin i \\
\left(\mathrm{~km} \mathrm{~s}^{-1}\right)\end{array}$ & $\begin{array}{c}M_{\text {acc }}\left(i=90^{\circ}\right) \\
\left(\mathrm{M}_{\odot}\right)\end{array}$ & $\begin{array}{c}M_{\text {acc }}\left(i=21^{\circ}\right) \\
\left(\mathrm{M}_{\odot}\right)\end{array}$ & $\begin{array}{c}\tau_{\min } \\
(\mathrm{yr})\end{array}$ \\
\hline BPS CS 22890-74 & G6V & 0.97 & 1.03 & $7 \pm 1$ & $0.0012 \pm 0.0002$ & $0.0034 \pm 0.0006$ & $3.2 \pm 0.6$ \\
HE 0430-2457 & K4V & 0.73 & 0.79 & $30 \pm 3$ & $0.0040 \pm 0.0005$ & $0.0111 \pm 0.0015$ & $13.7 \pm 2.4$ \\
J 02286-3625 & K0V & 0.87 & 0.93 & $90 \pm 5$ & $0.0141 \pm 0.0016$ & $0.0389 \pm 0.0043$ & $40.9 \pm 6.3$ \\
J 03154-5934 & F9V & 1.14 & 1.20 & $23 \pm 1$ & $0.0047 \pm 0.0005$ & $0.0129 \pm 0.0013$ & $10.6 \pm 1.5$ \\
J 03582-3609 & G0V & 1.08 & 1.14 & $30 \pm 2$ & $0.0057 \pm 0.0007$ & $0.0160 \pm 0.0019$ & $13.8 \pm 2.2$ \\
JL 277 & F5V & 1.33 & 1.39 & $33 \pm 2$ & $0.0077 \pm 0.0009$ & $0.0217 \pm 0.0026$ & $15.2 \pm 2.4$ \\
MCT 0146-2651 & G1V & 1.07 & 1.13 & $6 \pm 2$ & $0.0011 \pm 0.0004$ & $0.0032 \pm 0.0012$ & $2.7 \pm 1.0$ \\
PG 1514+034 & G6V & 0.97 & 1.03 & $18 \pm 2$ & $0.0031 \pm 0.0004$ & $0.0086 \pm 0.0013$ & $8.2 \pm 1.4$ \\
\hline
\end{tabular}

(Chen et al. 2013; Vos et al. 2015). Thus, if the current orbital period is shorter than about $1600 \mathrm{~d}$, it is likely that the systems was formed through stable RLOF.

There is no complete orbital coverage for the studied systems yet, but based on the RV amplitudes, the estimated periods of the systems discussed here are compatible with those of the already solved systems ( $P \approx 700-1300 \mathrm{~d}$; Vos et al. 2017). Therefore, it is very likely that these systems went through a mass-loss phase. The only alternative formation channel would be the double WD merger scenario in a triple system consisting of a close double WD binary in a wide orbit with an MS star, but it is unlikely for this scenario to be exclusively observed.

As the majority of the mass lost by the $\mathrm{sdB}$ progenitor is not accreted by the companion, it has to leave the system. This supports the proposed existence of circumbinary discs in wide $\mathrm{sdB}$ binaries. Based on binary evolution models, the sdB progenitor will lose more than $0.5 \mathrm{M}_{\odot}$, part of which might be lost through the outer Lagrange point (Vos et al. 2015). This mass will form a CB disc in which dust formation can take place. Photo-evaporation will remove the gas from the disc when the sdB star ignites its He core, but larger dust particles could survive for the duration of the core He-burning phase.

\section{CONCLUSIONS}

In this paper, we have presented a sample of confirmed composite sdB binaries containing 148 systems. This sample is constructed based on a literature review extended with systems from the GALEX and EC surveys, which were confirmed spectroscopically as composite sdB binaries. In many of these composite sdB binaries, the subdwarf component is formed through stable RLOF, resulting in a long-period binary with an MS companion. As this sample was constructed to study the post-RLOF population, all known shortperiod sdB binaries were removed. Based on observations with the UVES spectrograph, eight systems with an invalid composite sdB classification have been reclassified.

Based on the composite sdB sample, a long-term observing campaign of wide $\mathrm{sdB}$ binaries was started using the UVES at the VLT. No complete orbits have been obtained yet. However, for nine systems, enough spectra have been collected to create a master spectrum with a sufficiently high $\mathrm{S} / \mathrm{N}$ to perform a spectroscopic analysis of the cool companion and the sdB star.

The GSSP code used to derive the spectroscopic parameters of the cool companion was tested using three systems studied in the literature: $\mathrm{BD}+34^{\circ} 1543, \mathrm{BD}+29^{\circ} 3070$ and Feige 87 . The GSSP param- eters have been compared with parameters derived by independent methods, confirming the accuracy and precision of the GSSP code. This comparison shows that even though the contribution of the sdB component to the spectrum in GSSP is treated as a wavelengthindependent dilution factor, if the wavelength range is short enough, the parameters derived with GSSP for the cool companion are reliable.

The derived parameters of the nine cool companions in this paper show a diversity in spectral types, varying from mid $\mathrm{K}$ to mid $\mathrm{F}$, as well as a variety in metallicity from very metal poor to slightly super solar metallicity. A third interesting parameter is the rotational velocity of the companions, with slowly rotating stars at $v_{\mathrm{r}} \sin i<10 \mathrm{~km} \mathrm{~s}^{-1}$ to a fast rotator with $v_{\mathrm{r}} \sin i=90 \mathrm{~km} \mathrm{~s}^{-1}$.

The parameters for the cool companion derived with GSSP and with XTGRID match well within their error bars. Only the metallicity is an exception. For several of the systems, the metallicity derived with XTGRID is larger than the metallicity derived with GSSP. We consider that the most likely reason for this is the atmosphere models used by the two codes. This shows that when comparing metallicities of systems, it is important to know what atmosphere models were used to derive them.

The rotational velocity of the companions was used to estimate the amount and duration of accretion during the RLOF phase. By comparing the original synchronized rotation before RLOF to the observed rotational velocity, the amount of mass accretion can be estimated. Assuming that there is no significant angular momentum loss after the end of RLOF, we find that all systems have accreted very little mass, varying from $10^{-3}$ to $10^{-2} \mathrm{M}_{\odot}$. This is in agreement with the advanced evolutionary state of the companions in some wide sdB binaries (Vos et al. 2012). Such an evolution state could not have been reached if the companion had accreted a lot of mass during RLOF, as its original mass would have been too low to evolve off the MS. By using the maximum accretion rate based on the Eddington luminosity, the minimum accretion duration is found to be short, of the order of years. This lower limit does allow for the predicted thermal time-scale mass loss during RLOF from a red giant.

The nine systems analysed in this paper are part of a larger sample of $37 \mathrm{sdB}$ binaries that is currently being observed using UVES. The two methods that will be used to analyse these composite sdB binaries - grid search in stellar parameters (GSSP) and XTGRID have been described in detail. The orbital and spectral analysis of the full sample will provide a statistically viable sample to study the formation mechanisms of wide sdB binaries, and the necessary eccentricity pumping processes to explain the currently observed period-eccentricity distribution. 


\section{ACKNOWLEDGEMENTS}

This work is based on observations collected at the ESO, Chile, under programme IDs 088.D-0364, 093.D-0629, 096.D-0180, 097.D0110, 098.D-0018, 099.D-0014 and 099.D-0608. JV acknowledges financial support from FONDECYT in the form of grant number 3160504. This research has used the services of Astroserver.org under reference KW32YZ. The authors thank John Telting for observing J03397-2459 with the Nordic Optical Telescope. This publication makes use of data products from the 2MASS, which is a joint project of the University of Massachusetts and the Infrared Processing and Analysis Centre/California Institute of Technology, funded by the National Aeronautics and Space Administration (NASA) and the National Science Foundation. This research was made possible through the use of the AAVSO Photometric AllSky Survey (APASS), funded by the Robert Martin Ayers Sciences Fund. This research makes use of data products from the Galaxy Evolution Explorer (GALEX), which is a NASA Small Explorer (http://www.galex.caltech.edu). The mission was developed in cooperation with the Centre National d' Études Spatiales (CNES) of France and the Korean Ministry of Science and Technology.

\section{REFERENCES}

Aznar Cuadrado R., Jeffery C. S., 2001, A\&A, 368, 994

Barlow B. N., Wade R. A., Liss S. E., Østensen R. H., Van Winckel H., 2012, ApJ, 758, 58

Berger J., Fringant A.-M., 1980, A\&AS, 39, 39

Bertone E., Buzzoni A., Chávez M., Rodríguez-Merino L. H., 2004, AJ, 128,829

Bixler J. V., Bowyer S., Laget M., 1991, A\&A, 250, 370

Blanchette J.-P., Chayer P., Wesemael F., Fontaine G., Fontaine M., Dupuis J., Kruk J. W., Green E. M., 2008, ApJ, 678, 1329

Brassard P., Fontaine G., Billères M., Charpinet S., Liebert J., Saffer R. A., 2001, ApJ, 563, 1013

Brooker R. A., Olle T. W., 1955, MNRAS, 115, 101

Brown T. M., Ferguson H. C., Davidsen A. F., Dorman B., 1997, ApJ, 482, 685

Bruntt H., 2009, A\&A, 506, 235

Bruntt H. et al., 2002, A\&A, 389, 345

Bruntt H. et al., 2004, A\&A, 425, 683

Bruntt H., De Cat P., Aerts C., 2008, A\&A, 478, 487

Bruntt H. et al., 2010, MNRAS, 405, 1907

Chen X., Han Z., Deca J., Podsiadlowski P., 2013, MNRAS, 434, 186

Claret A., Gimenez A., 1989, A\&AS, 81, 37

Copperwheat C. M., Morales-Rueda L., Marsh T. R., Maxted P. F. L., Heber U., 2011, MNRAS, 415, 1381

Deca J. et al., 2012, MNRAS, 421, 2798

Degroote P. et al., 2011, A\&A, 536, A82

Demircan O., Kahraman G., 1991, Ap\&SS, 181, 313

Edelmann H., Heber U., Hagen H.-J., Lemke M., Dreizler S., Napiwotzki R., Engels D., 2003, A\&A, 400, 939

Freudling W., Romaniello M., Bramich D. M., Ballester P., Forchi V., GarcíaDabló C. E., Moehler S., Neeser M. J., 2013, A\&A, 559, A96

Geier S., 2013, A\&A, 549, A110

Geier S., Østensen R. H., Nemeth P., Gentile Fusillo N. P., Gänsicke B. T., Telting J. H., Green E. M., Schaffenroth J., 2017, A\&A, 600, A50

Green R. F., Schmidt M., Liebert J., 1986, ApJS, 61, 305

Green E. M., Liebert J., Saffer R. A., 2001, in Provencal J. L., Shipman H. L., MacDonald J., Goodchild S., eds, ASP Conf. Ser. Vol. 226, 12th European Workshop on White Dwarfs. Astron. Soc. Pac., San Francisco, p. 192 (arXiv:astro-ph/0012246)

Green E. M., Fontaine G., Hyde E. A., For B.-Q., Chayer P., 2008, in Heber U., Jeffery C. S., Napiwotzki R., eds, ASP Conf. Ser. Vol. 392, Hot Subdwarf Stars and Related Objects. Astron. Soc. Pac., San Francisco, p. 75
Greggio L., Renzini A., 1990, ApJ, 364, 35

Han Z., Tout C. A., Eggleton P. P., 2000, MNRAS, 319, 215

Han Z., Podsiadlowski P., Maxted P. F. L., Marsh T. R., Ivanova N., 2002, MNRAS, 336, 449

Han Z., Podsiadlowski P., Maxted P. F. L., Marsh T. R., 2003, MNRAS, 341, 669

Heber U., 1998, in Wamsteker W., Gonzalez Riestra R., Harris B., eds, ESA Special Publication Vol. 413, Ultraviolet Astrophysics Beyond the IUE Final Archive. ESA Publications Division, Noordwijk, p. 195

Heber U., 2016, PASP, 128, 082001

Heber U., Moehler S., Napiwotzki R., Thejll P., Green E. M., 2002, A\&A, 383, 938

Henden A. A., Templeton M., Terrell D., Smith T. C., Levine S., Welch D., 2016, VizieR Online Data Catalog, 2336

Hubeny I., Lanz T., 1995, ApJ, 439, 875

Hümmerich S., Bernhard K., Srdoc G., 2014, Open European Journal on Variable Stars, 167, 1

Husser T.-O., Wende-von Berg S., Dreizler S., Homeier D., Reiners A., Barman T., Hauschildt P. H., 2013, A\&A, 553, A6

Kawka A., Vennes S., O’Toole S., Németh P., Burton D., Kotze E., Buckley D. A. H., 2015, MNRAS, 450, 3514

Kilkenny D., Heber U., Drilling J. S., 1988, South African Astronomical Observatory Circular, 12

Koen C., Orosz J. A., Wade R. A., 1998, MNRAS, 300, 695

Kupfer T. et al., 2015, VizieR Online Data Catalog, 357

Lamontagne R., Demers S., Wesemael F., Fontaine G., Irwin M. J., 2000, AJ, 119, 241

Lubow S. H., Shu F. H., 1975, ApJ, 198, 383

Luo Y., Nemeth P., Liu C., Deng L., Han Z., 2016, ApJ, 818, 202

Martin D. C. et al., 2005, ApJ, 619, L1

Maxted P. F. L., Moran C. K. J., Marsh T. R., Gatti A. A., 2000, MNRAS, 311,877

Maxted P. f. L., Heber U., Marsh T. R., North R. C., 2001, MNRAS, 326, 1391

Moehler S., Richtler T., de Boer K. S., Dettmar R. J., Heber U., 1990, A\&AS, 86,53

Morales-Rueda L., Maxted P. F. L., Marsh T. R., North R. C., Heber U., 2003, MNRAS, 338, 752

Napiwotzki R., Karl C. A., Lisker T., Heber U., Christlieb N., Reimers D., Nelemans G., Homeier D., 2004, Ap\&SS, 291, 321

Németh P., Kawka A., Vennes S., 2012, MNRAS, 427, 2180

Østensen R. H., Van Winckel H., 2011, in Schmidtobreick L., Schreiber M. R., Tappert C., eds, ASP Conf. Ser. Vol. 447, Evolution of Compact Binaries. Astron. Soc. Pac., San Francisco, p. 171

Østensen R. H., Van Winckel H., 2012, in Kilkenny D., Jeffery C. S., Koen C., eds, ASP Conf. Ser. Vol. 452, Fifth Meeting on Hot Subdwarf Stars and Related Objects. Astron. Soc. Pac., San Francisco, p. 163 (arXiv:1112.0977)

O’Toole S. J., Heber U., 2006, A\&A, 452, 579

Paczynski B., 1976, in Eggleton P., Mitton S., Whelan J., eds, Proc. IAU Symp. Vol. 73, Structure and Evolution of Close Binary Systems. Kluwer, Dordrecht, p. 75

Pecaut M. J., Mamajek E. E., 2013, ApJS, 208, 9

Reed M. D., Stiening R., 2004, PASP, 116, 506

Ryabchikova T., Piskunov N., Kurucz R. L., Stempels H. C., Heiter U., Pakhomov Y., Barklem P. S., 2015, Phys. Scr, 90, 054005

Saffer R. A., Bergeron P., Koester D., Liebert J., 1994, ApJ, 432, 351

Shulyak D., Tsymbal V., Ryabchikova T., Stütz C., Weiss W. W., 2004, A\&A, 428, 993

Skrutskie M. F. et al., 2006, AJ, 131, 1163

Stobie R. S. et al., 1997, MNRAS, 287, 848

Tauris T. M., van den Heuvel E. P. J., 2006, in Lewin W., van der Klis M., eds, Compact Stellar X-ray Sources, Cambridge Astrophysics Series, No. 39. Cambridge University Press, Cambridge, p. 623

Tkachenko A., 2015, A\&A, 581, A129

Tsymbal V., 1996, in Adelman S. J., Kupka F., Weiss W. W., eds, ASP Conf. Ser. Vol. 108, M.A.S.S., Model Atmospheres and Spectrum Synthesis. Astron. Soc. Pac., San Francisco, p. 198 
Ulrich R. K., Burger H. L., 1976, ApJ, 206, 509

Vennes S., Kawka A., Németh P., 2011, MNRAS, 410, 2095

Vos J. et al., 2012, A\&A, 548, A6

Vos J., Østensen R. H., Németh P., Green E. M., Heber U., Van Winckel H., 2013, A\&A, 559, A54

Vos J., Østensen R., Van Winckel H., 2014, in van Grootel V., Green E., Fontaine G., Charpinet S., eds, ASP Conf. Ser. Vol. 481, 6th Meeting on Hot Subdwarf Stars and Related Objects. Astron. Soc. Pac., San Francisco, p. 265 (arXiv:1310.0189)
Vos J., Østensen R. H., Marchant P., Van Winckel H., 2015, A\&A, 579, A49 Vos J., Østensen R. H., Vučković M., Van Winckel H., 2017, A\&A, in press (arXiv:1706.02286)

Webbink R. F., 1984, ApJ, 277, 355

Zahn J.-P., 1977, A\&A, 57, 383

This paper has been typeset from a $\mathrm{T}_{\mathrm{E}} \mathrm{X} / \mathrm{L} \mathrm{T} \mathrm{E} \mathrm{X}$ file prepared by the author. 